\title{
The Exotic Eclipsing Nucleus of the Ring Planetary Nebula SuWt $2^{1}$
}

\author{
K. Exter, ${ }^{2}$ Howard E. Bond, ${ }^{3,4}$ K. G. Stassun ${ }^{5,6}$ B. Smalley, ${ }^{7}$ P. F. L. Maxted, ${ }^{7}$ and D. L. \\ Pollacco $^{8}$
}

Received —

\footnotetext{
${ }^{1}$ Based in part on observations obtained with the SMARTS Consortium 1.3- and 1.5m telescopes located at Cerro Tololo Inter-American Observatory, Chile, and with ESO Telescopes at the La Silla Observatory

${ }^{2}$ Space Telescope Science Institute, 3700 San Martin Dr., Baltimore, MD 21218, USA. Current address: Institute voor Sterrenkunde, Katholieke Universiteit Leuven, Leuven, Belgium; katrina@ster.kuleuven.be

${ }^{3}$ Space Telescope Science Institute, 3700 San Martin Dr., Baltimore, MD 21218, USA; bond@stsci.edu

${ }^{4}$ Visiting astronomer, Cerro Tololo Inter-American Observatory, National Optical Astronomy Observatory, which is operated by the Association of Universities for Research in Astronomy, under contract with the National Science Foundation. Guest Observer with the International Ultraviolet Explorer, operated by the Goddard Space Flight Center, National Aeronautics and Space Administration.

${ }^{5}$ Dept. of Physics and Astronomy, Vanderbilt University, Nashville, TN 32735, USA

${ }^{6}$ Department of Physics, Fisk University, 1000 17th Ave. N, Nashville, TN 37208, USA

${ }^{7}$ Astrophysics Group, Chemistry \& Physics, Keele University, Staffordshire, ST5 5BG, UK

${ }^{8}$ Queen's University Belfast, Belfast, UK
} 


\begin{abstract}
SuWt 2 is a planetary nebula $(\mathrm{PN})$ consisting of a bright ionized thin ring seen nearly edge-on, with much fainter bipolar lobes extending perpendicularly to the ring. It has a bright (12th-mag) central star, too cool to ionize the PN, which we discovered in the early 1990's to be an eclipsing binary. Although it was anticipated that there would also be an optically faint, hot, ionizing star in the system, a spectrum from the International Ultraviolet Explorer (IUE) did not reveal a UV source. We present extensive ground-based photometry and spectroscopy of the central binary collected over the ensuing two decades, resulting in the determination that the orbital period of the eclipsing pair is 4.9 days, and that it consists of two nearly identical A1 V stars, each of mass $\sim 2.7 M_{\odot}$

The physical parameters of the A stars, combined with evolutionary tracks, show that both are in the short-lived "blue-hook" evolutionary phase that occurs between the main sequence and the Hertzsprung gap, and that the age of the system is about 520 Myr. One puzzle is that the stars' rotational velocities are different from each other, and considerably slower than synchronous with the orbital period. It is possible that the center-of-mass velocity of the eclipsing pair is varying with time, suggesting that there is an unseen third orbiting body in the system. We propose a scenario in which the system began as a hierarchical triple, consisting of a $\sim 2.9 M_{\odot}$ star orbiting the close pair of A stars. Upon reaching the AGB stage, the primary engulfed the pair into a common envelope, leading to a rapid contraction of the orbit and catastrophic ejection of the envelope into the orbital plane. In this picture, the exposed core of the initial primary is now a white dwarf of $\sim 0.7 M_{\odot}$, orbiting the eclipsing pair, which has already cooled below the detectability possible by $I U E$ at our derived distance of $2.3 \mathrm{kpc}$ and a
\end{abstract}


reddening of $E(B-V)=0.40$. The SuWt 2 system may be destined to perish as a Type Ia supernova.

Subject headings: binaries: eclipsing — stars: individual (NSV 19992) — planetary nebulae: individual (SuWt 2) 


\section{Introduction}

Near the ends of their lives, many, or possibly all, low- and intermediate-mass stars eject the tenuous outer layers they had developed on the asymptotic giant branch (AGB), evolve rapidly to high surface temperature, and ionize the surrounding ejecta, producing a planetary nebula $(\mathrm{PN})$. The detailed processes of nebular ejection remain uncertain; however, over the past few decades, it has become clear that binary-star interactions play a role in the production of a significant fraction of PNe. The evidence supporting this view includes (1) approximately 10-20\% of planetary-nebula nuclei (PNNi) are photometrically variable, and sometimes eclipsing, binaries with short orbital periods of hours to a few days (Bond \& Livio 1990; Bond 2000; Miszalski et al. 2008a, 2009; and references therein);

(2) among a small sample of bright PNNi, nearly all showed short-timescale radial-velocity variability (De Marco et al. 2004), suggestive of membership in close binaries; and (3) the prevalence of non-spherical morphologies among PNe. While an axisymmetric morphology can easily result if ejection is shaped, or indeed directly caused by, binary interactions, alternative models that have successfully recreated these morphologies include magnetized winds and rapid stellar rotation (cf. Dobrinĉić et al. 2008 and the reviews of Balick \& Frank 2002 and Kwok 2008). In addition, recent deep searches for faint PNe have shown that the fraction having spherical morphologies is higher among low-surface-brightness nebulae (e.g., Parker et al. 2006; Miszalski et al. 2008b; Jacoby et al. 2010); hence the number of known PNe that do not necessarily require a binary central system is larger now than before these deep searches. Thus the degree to which binary-star ejection is a major channel for the production of PNe remains uncertain and controversial; see the reviews by Bond (2000) and De Marco (2009).

In this paper we discuss the properties of the eclipsing binary central star of the PN SuWt 2, based on observations collected by us over the past two decades. This object 
presents a number of challenges to our understanding, as described above, of PNe with close-binary nuclei. We have presented several unrefereed progress reports on SuWt 2 in past years as we gradually learned more about the system (Bond, Ciardullo, \& Webbink 1996; Bond, Exter, \& Pollacco 2001; Bond et al. 2002; Exter et al. 2003; Bond et al. 2008); in this journal paper we now discuss our extensive material on this remarkable object in detail.

\section{2. $\mathrm{SuWt} 2$}

The PN SuWt 2 (PN G311.0+02.4) was detected by Schuster \& West (1976) on deep ESO 1-m Schmidt plates taken during emulsion-sensitization tests. (The nebula had actually been discovered earlier by Andrews \& Lindsay 1967 on long-exposure plates taken with the ADH Baker-Schmidt telescope, but to our knowledge this prior discovery has not been referenced in the literature until now.) Schuster \& West described the nebula as an elliptical ring enclosing a prominent and relatively blue central star. Follow-up image-tube spectrograms obtained by West (1976) confirmed the emission-line PN nature of the nebula, with a heliocentric nebular radial velocity of $-40 \pm 9 \mathrm{~km} \mathrm{~s}^{-1}$. West quotes a private communication from N. Sanduleak, who classified the central star B9 V from a Curtis Schmidt objective-prism plate (below, from more modern data, we slightly revise the spectral type to early A).

Narrow-band CCD images of SuWt 2 were presented by Schwarz, Corradi, \& Melnick (1992). The angular dimensions of the bright ring, based on these images, are $86 . .5 \times 43^{\prime \prime} .4$ (Tylenda et al. 2003). Further deep imaging and spectroscopy has been obtained by Smith, Bally, \& Walawender (2007) and Jones et al. (2009), and these images show faint bipolar lobes extending along an axis perpendicular to the plane of the brighter ring. This structure is also seen in the SuperCOSMOS H $\alpha$ image available online (Parker et al. 2005). Jones et 
al. give a nebular systemic radial velocity of $-25 \pm 5 \mathrm{~km} \mathrm{~s}^{-1}$, and present very deep images of the nebula. To illustrate the extended, low-surface-brightness lobes in a wider field of view than shown by Jones et al., we present in Fig. 1 a deep image in an $\mathrm{H} \alpha+[\mathrm{N}$ II] filter, obtained by H.E.B. on 1995 January 31 with the CTIO 1.5-m telescope1.

The elliptical ring exhibited by SuWt 2 is suggestive of a thin annulus viewed at a high inclination. Assuming the ring to be perfectly circular, the viewing inclination implied by the axis ratios measured by Smith et al. (2007) is $i=64^{\circ} \pm 2^{\circ}$, or is $68^{\circ} \pm 2^{\circ}$ for the proportions measured by Jones et al. (2009). The bright, hollow, thin, and nearly perfectly elliptical ring, with considerably fainter bipolar lobes, is an unusual morphology among PNe. However, a morphologically very similar object is the northern ring PN WeBo 1. Its optical central star is a cool barium giant, which is photometrically variable with a period of 4.7 d (Bond, Webbink, \& Pollacco 2003); however, unlike the eclipsing SuWt 2, the variability of WeBo 1's nucleus is believed to be due to starspot activity on the rotating cool star. Another PN with a similar thin elliptical ring structure is SuWt 3 (West \& Schuster 1980), but its central star is very faint. In a separate context, thin nebular rings are known around several hot, massive supergiants, as well as SN 1987A, and have been discussed extensively (Smith et al. 2007 and references therein).

The B9 V spectral type of the star (as reported at the time of the discovery of the PN) at the center of the SuWt 2 nebula indicated an effective temperature too cool to ionize the nebula. This, along with the PN's morphology, suggested that there is likely to be an additional star in the system, smaller and optically fainter than the optical component, but hot enough to emit ionizing flux. These considerations, along with a morphology suggestive of ejection into an equatorial plane viewed at high inclination, made the optically bright

\footnotetext{
${ }^{1}$ A pseudo-color version of this image, made from frames in [O III] $5007 \AA$ and $\mathrm{H} \alpha+[\mathrm{N}$ II], is available at http://hubblesite.org/newscenter/archive/releases/2008/21/image/a/
} 
star in SuWt 2 a prime candidate for a program of photometric monitoring of PNNi aimed at discovering close binaries.

Several measurements by H.E.B. during an observing run in 1990 at the 0.9-m telescope at the Cerro Tololo Inter-American Observatory (CTIO) disclosed no light variations. However, a 13-night CCD observing run on the same telescope in 1991 resulted in the discovery of two eclipses, separated by 5 days. Continued monitoring during subsequent observing runs in the 1990s led to the finding that the eclipses recur every 2.5 days. Thus the system contains either a hot and a cool component (as is typical of the other known eclipsing $\mathrm{PNNi}$ ), in an orbital period of 2.5 days with one deep eclipse per orbit, or two stars of similar temperature, with an orbital period of 5 days and two deep eclipses per orbit. Due to the lack of any obvious reflection-effect variability outside the eclipses, indicating that heating effects are insignificant, the latter possibility seemed more likely. Subsequent high-S/N (signal-to-noise) AAT echelle spectroscopic observations (see below) in fact revealed a double-lined system containing two nearly equal-brightness stars, confirming that the period is indeed close to 5 days and that there are two eclipses of comparable depth per orbital period.

In this paper we assemble and analyze the photometry and optical and UV spectroscopy that we have obtained over the past two decades. The result is a determination of the stellar and binary parameters for the eclipsing system, along with the raising of a number of intriguing astrophysical puzzles.

In the discussion below we use "SuWt 2" to refer to the PN; for the central star, which has not yet received a formal variable-star designation, we use "NSV 19992", which is the star's number in the New Catalogue of Suspected Variable Stars (Kazarovets, Samus, \& Durlevich 1998). 


\section{Photometry}

Because the orbital period of NSV 19992 is very close to 5 days, with an eclipse duration of more than 17 hours, accumulation of a light curve covering all orbital phases has been a slow process, involving several different telescopes. Most of our photometry is differential with respect to several nearby comparison stars, but on several photometric nights we also measured absolute photometry. Stringent checks were carried out to ensure that the various data sets could be combined onto the same system.

Our photometric observations have all been made with a variety of CCD cameras on telescopes at CTIO. Data were obtained with the 0.9 - and $1.5-\mathrm{m}$ reflectors on 63 nights between 1990 and 2001. In 2003 the small- and medium-size telescopes at CTIO came

under the management of the Small and Medium-Aperture Research Telescopes System (SMARTS) Consortium, and we used the SMARTS 1.3-m telescope in 2007 for additional observations on 11 nights, as described below.

A finding chart identifying NSV 19992 and three nearby comparison stars that we selected, designated C1, C2, and C3, is given in Fig. 2. Standard data reduction-bias subtraction and flat-fielding - has been carried out by us, or for the SMARTS observations by the automated pipeline at Yale University.

\subsection{Absolute Calibration}

Absolute photometry of NSV 19992 and the comparison stars was obtained with the CTIO 0.9- and 1.5-m telescopes and the SMARTS 1.3-m telescope on 10 photometric nights between 1990 and 2007, with a total of 14 individual BVI observations. Photometric reductions were performed using the DAOPHOT II package (Stetson 1987, 2000) running 
under IRAF 2 or with simple aperture photometry. Calibration to the standard JohnsonKron-Cousins BVI system was done using observations of standard fields from Landolt (1992) and transformation routines in the IRAF PHOTCAL package.

The calibrated magnitudes and colors for NSV 19992 and the three comparison stars are given in Table 1, along with the stars' coordinates from the USNO NOMAD astrometric catalog (Zacharias et al. 2004). We saw no evidence for variability of the comparison stars, nor of the outside-eclipse brightness of NSV 19992 itself, above a level of 0.02 mag during this 17-year interval.

\subsection{Differential Photometry}

CCD differential photometry of NSV 19992 and the three comparison stars in the $B V I$ filters was taken with the CTIO and SMARTS telescopes in various runs between 1990 and 2007. During the later years, as the eclipse ephemeris became better known, it became possible to observe several eclipses intensively during entire nights. We took advantage of STScI's membership in the SMARTS Consortium by obtaining individual service observations of NSV 19992 at critical orbital phases on 11 nights in 2007 April and June, thus filling in phases that still had not been covered in the earlier runs, as well as improving the precision of the orbital ephemeris.

Instrumental magnitudes were obtained using the IRAF APPHOT aperture-photometry routine and manual aperture photometry using IMEXAMINE, and PSF fitting magnitudes were obtained using DAOPHOT II running under IRAF.

\footnotetext{
${ }^{2}$ IRAF is distributed by the National Optical Astronomy Observatory, which is operated by the Association of Universities for Research in Astronomy (AURA) under cooperative agreement with the National Science Foundation.
} 
As shown in Table 1, the comparison stars C1 and C2 are significantly redder than NSV 19992; this introduced offsets into the raw differential photometry because of variations in the effective wavelengths of the bandpasses with the different cameras and CCDs used over the years, particularly in the $B$ band. In our final reductions, we used differential magnitudes with respect only to C3, which has similar colors to NSV 19992. Color-dependent corrections were made to the differential measurements based on the transformation equations derived from the standard stars, and then the adjusted differential magnitudes were added to the calibrated values for C3 listed in Table 1, to place the final photometry of NSV 19992 on the standard BVI system. Because of the similarity of colors between NSV 19992 and C3, we did not correct for trends with airmass. Complete tables of our photometry will be lodged with CDS3.

\subsection{Orbital Ephemeris and Light Curve}

Portions of eclipses of sufficient duration for determining times of minimum were observed during long runs between 1997 and 2007. We measured the times of minimum by fitting a Gaussian to the data points around each eclipse for each filter, with the fitting errors being used to determine formal uncertainties in the resulting eclipse times. Then, knowing already that the period was close to 5 days, we assigned cycle counts to each eclipse (two of which are secondary eclipses), and finally performed a weighted least-squares fit using a linear ephemeris to solve for the orbital period $P$ and time of primary eclipse $T_{0}$. The times of eclipse, cycle counts, residuals, and filters used are given in Table 2 ,

The final adopted ephemeris is

$$
\text { Pri. Min. }(\mathrm{HJD})=2450668.5915(10)+4.9098505(20) E \text {. }
$$

\footnotetext{
${ }^{3}$ http://cdsweb.u-strasbg.fr/astroWeb/astroweb.html
} 
Using the Lafler-Kinman (1965) algorithm, we confirmed that the adopted period, within the quoted uncertainty, fits the entirety of our data from 1990 to 2007.

All the 280 BVI data points were then phased to the ephemeris to produce the light and color curves shown in Fig. 3. On photometric nights the Poissonian errors are less than \pm 0.01 mag, i.e., smaller than the plotting symbols in the figure. However, several of the eclipses were observed on non-photometric and even cloudy nights, producing a larger photometric scatter under those conditions. There are also some small residual systematic differences remaining between the data from different cameras and telescopes, of an uncertain origin (possibly flat-fielding or vignetting issues), at a level of about 0.02 mag. These account, for example, for the apparent small excursions just before the eclipse ingresses in Fig. 3.

The colors are essentially constant throughout both eclipses, and the eclipses have nearly identical depths; both facts are consistent with the two stars having similar effective temperatures. The primary eclipse, at orbital phase 0 , is actually very slightly deeper than the secondary eclipse, and as we will show below the primary star eclipsed at phase 0 is slightly hotter and slightly more massive than the secondary component, and is a faster rotator. We marginally detect the light variations due to the slightly ellipsoidal shapes of the stars, giving rise to small increases in brightness around phases 0.25 and 0.75 . The secondary eclipse occurs at phase 0.5 , so there is no evidence for a non-zero orbital eccentricity.

\section{Stellar Spectroscopy}

Here we discuss a range of spectroscopic observations of NSV 19992: in the ultraviolet using the International Ultraviolet Explorer (IUE), for the purpose of searching for a hot 
companion of the optical central binary (of two nearly identical A stars) and for obtaining the UV spectral energy distribution (SED); ground-based spectroscopy for the purpose of measuring radial velocities of the binary; and ground-based spectroscopy for the purpose of a spectral-type analysis and determination of the optical SED. The reduction of all data followed the standard scheme (bias subtraction, flat-fielding, sky subtraction), followed by conversion of the spectra to a constant velocity grid, correction to the heliocentric standard, and a continuum normalization from a low-order polynomial fit. We used the IRAF and Starlink 4 environments for the data reduction, and MOLLY and PAMELA 5 were used for the wavelength calibrations and velocity normalizations. Arc spectra generally bracketed the targeted pointings. Details of the observations and data reduction are given in Table 3, and below we describe only those details unique to each run.

\subsection{International Ultraviolet Explorer}

As part of a program of UV spectroscopy of optically bright PNNi that appear to be too cool to ionize their nebulae, M. Meakes and H.E.B. observed SuWt 2 with IUE in 1990 and 1991. The first short-wavelength low-dispersion spectrum was expected to reveal the UV spectrum of a hot PN central star component; however, the spectrum showed only a faint short-wavelength continuum, which is compatible with that expected from the A-type components of NSV 19992. The 1991 short- and long-wavelength spectra again only showed the continuum of NSV 19992, whose flux drops to a nearly undetectable level below $\sim 1500 \AA$. The IUE results were described briefly by Bond et al. (2002). We return to the IUE spectra below in $§ 9.4$.

\footnotetext{
${ }^{4}$ http://starlink.jach.hawaii.edu

${ }^{5}$ MOLLY and PAMELA are software written by Tom Marsh, Warwick, UK
} 
In retrospect, we see that the IUE observations were made at orbital phases of 0.94 , 0.68 , and 0.70 , respectively; thus they were all taken outside the eclipses of the pair of A stars.

\subsection{SAAO}

NSV 19992 was observed in 1995 (by D.L.P. and F. Marang) with the SAAO 1.9-m and the RPCS (Reticon Photon Counting System). The RPCS was an instrument that produced two $1 \times 1800$-pixel spectra: one of NSV 19992 and one of the sky. No nebular lines were visible on the target or sky spectra. The continuum signal-to-noise $(\mathrm{S} / \mathrm{N})$ ratio

for these spectra is around 28. Analysis of the velocity standard stars observed on the same nights shows there are no drifts in the radial-velocity scale over the observing run, with a scatter about measured versus true radial velocity of $\pm 6 \mathrm{~km} \mathrm{~s}^{-1}$.

\subsection{NTT}

NSV 19992 was observed in 1995 with EMMI on the 3.5-m ESO New Technology Telescope (NTT) at La Silla under remote control from Garching, Germany. We used a slit 1" wide to ensure radial-velocity accuracy. The spectra were "optimally extracted," i.e., weighted to give the maximum S/N ratio, of up to 100 in the continuum. No nebular lines were visible on the CCD images. No radial-velocity standard stars were observed during this run. 


\section{4. $\mathrm{AAT}$}

NSV 19992 was observed with the UCLES echelle spectrograph on the 3.9-m AAT in 2000. These nights suffered from scattered clouds and had an average seeing of 1 ". 8 . The continuum $\mathrm{S} / \mathrm{N}$ for these spectra is around 60 . No radial-velocity standards were observed during the run.

These high-resolution AAT echelle spectra are shown in Fig. 4; these firmly confirmed for the first time that the spectrum is double-lined, consisting of two A-type stars, and thus established that the 4.9-day period was the correct one.

Additional data were taken with the RGO spectrograph of the AAT in 2001. The slit width was set to match the seeing. The continuum S/N ratio for these spectra is around 50 . Analysis of radial-velocity standards observed during the run resulted in nightly corrections of $<2 \mathrm{~km} \mathrm{~s}^{-1}$.

\subsection{CTIO and SMARTS}

Spectra of NSV 19992 were obtained by H.E.B. with the Ritchey-Chretien (RC) spectrograph on the CTIO 1.5-m in 2001 and 2003 (at which time the telescope had come under management by the SMARTS consortium), and by SMARTS service observers in 2006 and 2007. There were no changes in the instrumentation over the 2001-2007 interval. The slit width was $2^{\prime \prime}$ for the former two years and $3^{\prime \prime}$ for the latter two. Nebular contamination of the stellar spectra at the Balmer lines was obvious for these data, and was removed using the spectra from the nebular regions close to the star on the CCD. Flux-calibration standards (Hiltner 60 and LTT 4364) were observed for all these observations. The reduction of these data hence included flux calibration, with a scatter in the sensitivity function of $\pm 0.01 \mathrm{mag}$. Note that slit losses were not corrected for, and we are uncertain if the 
conditions were truly photometric. Therefore, we cannot guarantee the absolute flux scale is correct.

\section{Spectral Analysis}

\subsection{Spectral Energy Distribution}

We have compiled the SED of NSV 19992 from the UV to the NIR by combining data from several sources. The UV fluxes were obtained from the low-resolution IUE spectra

described in $§ 4.1$. The spectra were binned into $50 \AA$ regions, and only wavelength ranges with $\mathrm{S} / \mathrm{N}>10$ were retained. In the optical region we used the broad-band magnitudes given in Table 1 and the flux-calibrated spectra (§4.5). For the NIR we used broad-band photometry from the DENIS and 2MASS surveys (listed in Table 1).

We then fit reddened Kurucz (1993) model flux distributions to the SED. The flux distribution is well fitted using a single-star model, as expected because of the close similarity of the two stars implied by the light curve. The best fit was obtained with an effective temperature of $T_{\text {eff }}=9500 \pm 500 \mathrm{~K}$, a surface gravity of $\log g=4.0 \pm 0.5$, and reddening of $E(B-V)=0.40 \pm 0.05$. The combined energy distribution is shown in Fig. 5 , along with the best-fitting Kurucz model. Direct integration of the observed flux distribution gives $f_{\oplus}=(1.25 \pm 0.20) \times 10^{-9} \mathrm{ergs} \mathrm{s}^{-1} \mathrm{~cm}^{-2}$, corrected for $E(B-V)=0.40$. The Infrared Flux Method (Blackwell \& Shallis 1977) gives a similar result of $T_{\text {eff }}=9430 \pm 560 \mathrm{~K}$ and a combined angular diameter of the two stars of $\theta=0.022 \pm 0.003$ mas.

The corresponding spectral types for both stars would be near A1 V. The dereddened color of the combined light is $(B-V)_{0}=0.02$, consistent with the spectral type. 


\subsection{Spectral Diagnostics and Rotational Velocities}

As described below in $\$ 6.1 .1$, from the AAT echelle spectra we disentangled the spectral lines from the two components of the binary into spectra of the individual stars. From the individual spectra we measured equivalent widths of several unblended lines in the 4460-4500 A range. These were used with the LTE spectrum-synthesis code UCLSYN (Smith 1992; Smalley, Smith, \& Dworetsky 2001) to determine stellar parameters and chemical abundances.

The parameter space around that indicated by the SED analysis described above was searched to find the best self-consistent solution for (i) the Fe II/Fe I ionization balance, (ii) the microturbulent velocity $\left(\xi_{\mathrm{t}}\right)$ from the Ti II lines, and (iii) a null-dependence of the results on excitation potential. The resulting stellar parameters for the components of NSV 19992 and their chemical abundances are given in Table 4. The star we have designated as the primary is marginally hotter than the secondary, but the difference is not statistically significant.

Plots of the model fits to the $\mathrm{Mg}$ II $4481 \AA$ and $\mathrm{H} \alpha$ lines of the echelle spectra are shown in Fig.6.

We fitted the $v \sin i$ of both stars while running UCLSYN, assuming an instrumental FWHM of $0.14 \AA$. The lines of the primary star are clearly rotationally broadened, with $v \sin i=17 \mathrm{~km} \mathrm{~s}^{-1}$; in the discussion below we sometimes refer to the primary as the "fast" rotator. For the secondary star we only obtain an upper limit to $v \sin i$ of $5 \mathrm{~km} \mathrm{~s}^{-1}$. The derived value of $v \sin i$ is not strongly dependent on the adopted physical parameters. The

luminosity ratio was calculated at $4480 \AA$, and is not significantly different from equal luminosities of the two stars.

There is a hint that the He I $4471 \AA$ line is present in the slow secondary, but not 
in the fast primary. However, the $\mathrm{S} / \mathrm{N}$ ratio is rather poor for this faint feature and the identification of He I in the secondary star is uncertain. The Mg II $4481 \AA$ line was difficult to fit with a solar abundance, but a value of $[\mathrm{Mg} / \mathrm{H}] \simeq+0.4$ dex gives a good fit to the observed line profiles. The Mg II 4481 line is known to exhibit marked deviations from LTE (Przybilla et al. 2001), which could account for the apparent overabundance. A value of $[\mathrm{Ti} / \mathrm{H}]$ of $+0.59 \pm 0.15$ was formally derived for the primary star. However, we consider this finding to be more uncertain than the errors reflect; NLTE effects may be important.

\section{Spectroscopic Orbit}

From our high-resolution (UCLES, RGO, RPCS) spectra we measured the radial velocities of the two stars in the binary, leading to a determination of the orbital parameters and the stellar masses. There was some interplay between the spectroscopic and photometric analyses, allowing a narrowing of the range of possibilities based on the other's results.

\subsection{Measuring the Radial Velocities}

The AAT echelle UCLES spectra were the most important for fitting the radial velocities with a binary orbit model, as they are at excellent velocity resolution. Their phase coverage, however, is a little sparse, and this is where the AAT RGO mid-resolution spectra become useful. The SAAO RPCS lower-resolution spectra were also useful, despite the low velocity precision and lower SNR, because they have good orbital coverage; it is also the case that as they were taken 5 years before the echelle spectra they could be used to check for any drift in the systemic velocity of NSV 19992 (for example, as expected if a third star were present in the system). To this particular study we have added three low-resolution spectra taken with the NTT. 
All velocities were corrected to the heliocentric frame. The AAT mid-resolution and SAAO data have been checked for velocity offsets using observations of radial-velocity standards. In Table 5 we list the velocities, and next we describe how they were measured.

\subsubsection{Cross-Correlation for the AAT Echelle Spectra}

Radial velocities were determined from the high-resolution echelle spectra using cross-correlation with a template spectrum. The template spectrum was created from those which we modeled for each star of the binary. The main difference between the two stars is their rotational velocities, and this was accounted for in the templates.

The cross-correlation functions (CCFs) show two very distinct peaks, which are clearly resolved in all but one case. To measure the position of the peaks in the CCFs and check that the rotational broadening that had been applied to the spectra was sufficient, we calculated two model CCFs of the synthetic spectrum (one for each star) against two versions of itself after convolution with the rotational broadening function of Unsöld (1955). The broadening necessary for each star are consistent with the values found already: $15 \mathrm{~km} \mathrm{~s}^{-1}$ for the fast rotator and none for the slow rotator. We then used a least-squares method to optimize the fit to the CCFs using the sum of the two model CCFs. The free parameters for the fit are the radial-velocity shift between the model CCF and the star and a scaling factor for each CCF. An example of one such fit is shown in Fig. 7. Also shown are the echelle spectra from each component after being "disentangled" (Hynes \& Maxted 1998), revealing each component's spectrum alone. 


\subsubsection{Gaussian Line Fitting}

Gaussian fitting of individual absorption lines was used to measure the radial velocities from the lower-resolution AAT and SAAO spectra. For this we focussed on the Mg II $4481 \AA$ line and did the fitting in the MOLLY environment. We determined the wavelength of the $\mathrm{Mg}$ II feature from the theoretical spectra for each star (\$5), broadened and resampled to match the AAT and SAAO spectra $\left(\lambda_{0}=4481.2195 \AA\right.$ in all cases $)$. We also fit to the Balmer lines at the phases around quadrature, where the lines from each star somewhat separate on the observed spectra, but only as a check on the superior Mg II results.

The theoretical spectra used in the cross-correlation (the echelle spectra) are of zero velocity, so for these the systemic velocity (that of the whole binary with respect to us) should be on the correct scale. To ensure that the Gaussian-fit velocities (the mid-resolution spectra) were on the same scale we compared the cross-correlation results for the echelle spectra with Gaussian-fit results for the echelle spectra, from which we found a value $3.3 \mathrm{~km} \mathrm{~s}^{-1}$ to be subtracted from the Gaussian-fit velocities.

\subsection{Orbit Solution}

We used the double-lined spectroscopic binary orbital-solution code BINARY 6 developed by Gudehus (2001), to fit for the orbital parameters of NSV 19992. We fitted each observing run of radial-velocity measurements separately in order to account for possible shifts in the center-of-mass velocity of the system with time. These possible shifts are discussed again in $\S 7.3$.

We began by fitting the radial velocities determined from the AAT echelle data obtained

\footnotetext{
${ }^{6}$ available at http://www. chara.gsu.edu/\$^\{\scriptscriptstyle \sim\}\$gudehus/binary.html
} 
in 2000, as these are the highest-resolution spectra and thus yield the highest-precision radial-velocity measurements. We held the orbital period fixed to the already-determined ephemeris (Eq. 1), but fit for all other orbital parameters. This fit formally yields a slight, and marginally significant, non-zero eccentricity of $e=0.023 \pm 0.011$. However, as noted at the end of $\S 3.3$, the light-curve eclipse times show that the secondary eclipses occur at phase 0.5 (see also the $O-C$ residuals of the eclipse timings in Table 2), giving no support for a non-zero eccentricity. Moreover, the eccentric orbit solution predicts eclipse times that are inconsistent with the observed eclipse times. From our data we cannot fix more accurate limits for the eccentricity; hence for this paper we fix it to a value of 0.0. We refit the AAT echelle radial-velocity data with $e=0$ and obtained the orbital parameters listed in Table 6. A follow-up study of the small but possibly non-zero eccentricity could add insight into the history of this system.

Next, we fit each of the other three epochs of radial-velocity data (two runs in 1995, one in 2001) by fixing all orbital parameters to the values determined from the above fit, save for the center-of-mass velocity, $v_{\gamma}$. We did this to look for any systemic velocity offset of each epoch relative to the AAT echelle epoch. The resulting best-fit systemic velocities are tabulated in Table 7, where indeed we see evidence for systemic velocity shifts of up to $\sim 40 \mathrm{~km} \mathrm{~s}^{-1}$. We discuss these systemic velocity shifts in the context of a possible third body in the NSV 19992 system below (\$6.3).

We subtracted the best-fit $v_{\gamma}$ from each epoch and performed a final fit to all of the radial-velocity data together. The resulting orbit parameters are summarized in Table 8 and the fit is displayed in Fig. 8 .

The components of the NSV 19992 eclipsing binary evidently have nearly identical masses. The formally more massive primary $\left(M_{1}\right)$ is identified as the rapid rotator (i.e., the "fast" star). We emphasize, however, that the best-fit mass ratio of 
$q=M_{2} / M_{1}=0.9922 \pm 0.0044$ (Table 9 ) is consistent with unity at the $\approx 2 \sigma$ level and thus we cannot definitively establish which component is truly the more massive one. As we discuss in $\$ 9.1$, the rapid evolutionary timescales of the components make it probable that the true mass ratio is nearly exactly 1.0.

\subsection{Evidence for a Third Body}

As noted above, we observe changes in the systemic velocity from one epoch to the next: $-43 \mathrm{~km} \mathrm{~s}^{-1}$ in $1995.3,-21 \mathrm{~km} \mathrm{~s}^{-1}$ in $1995.4,-8 \mathrm{~km} \mathrm{~s}^{-1}$ in 2000.4 , and $-4 \mathrm{~km} \mathrm{~s}^{-1}$ in 2001.2. The first value is based on an observational study of only three spectra with no radial-velocity standards also observed. Hence its uncertainty is larger than the formal error. We address this in the discussion that follows.

These different systemic velocity values suggest that the NSV 19992 eclipsing binary (EB) itself orbits a third body. With only four distinct epochs it is impossible to determine the period or amplitude of these variations. However, the rapid change observed between the 1995.3 NTT and 1995.4 SAAO data, separated by only $0.10 \mathrm{yr}$, suggests a relatively short-period orbit, of order a few months. If we assume that the observed range of $v_{\gamma}$ values of about $40 \mathrm{~km} \mathrm{~s}^{-1}$ represents the extremes of the EB's orbit about the putative third body, then the mean systemic velocity of NSV 19992 is near $-23 \mathrm{~km} \mathrm{~s}^{-1}$ (assuming a circular orbit), consistent with the nebular radial velocity of $-25 \pm 5 \mathrm{~km} \mathrm{~s}^{-1}$ (Jones et al. 2009).

If we further assume that the time between the NTT and SAAO data epochs (1995.3 and 1995.4) represents $\sim 1 / 2$ of the orbital period about the third body, this orbit can be characterized by a period of $P_{3} \approx 0.2 \mathrm{yr}$ and a velocity semi-amplitude of $K_{\mathrm{EB}} \approx 20 \mathrm{~km} \mathrm{~s}^{-1}$. The mass function for the third body is then given by

$$
f(M)=\frac{M_{3}^{3} \sin ^{3} i_{3}}{\left(M_{3}+M_{\mathrm{EB}}\right)^{2}}=0.061\left(\frac{K_{\mathrm{EB}}}{20 \mathrm{~km} \mathrm{~s}^{-1}}\right)^{3}\left(\frac{P_{3}}{0.2 \mathrm{yr}}\right) M_{\odot},
$$


where $M_{3}$ is the mass of the third body, $M_{\mathrm{EB}}=M_{1}+M_{2}$ is the total mass of the EB ( $\sim 5.4 M_{\odot}$ according to the previous subsection), and $i_{3}$ is the inclination of the third body's orbit, assumed to be circular. In the evolutionary scenario that we outline in $\S 9.5$ the system is a triple in which the initially more massive star has formed the PN and so it should be orbiting in the plane of the nebular ring, which is viewed at an inclination of $\sim 68^{\circ}$. Thus we expect $\sin i_{3} \approx 0.9$.

To yield the above $f(M)$ therefore requires an unseen mass $\sim 1.54 M_{\odot}$. Even if the orbit is viewed edge-on, the mass is about $1.41 M_{\odot}$. Our result is problematic, not only because it is at or above the Chandrasekhar limit, but also because the evolutionary scenario proposed below $(\S 9.5)$ - in which the third body was originally a slightly more massive star of $\sim 2.9 M_{\odot}$-requires a considerably lower remnant mass, of about $0.7 M_{\odot}$.

The discrepancy would be alleviated if we gave low weight to the outlying systemic velocity from the low-resolution NTT spectra of 1995; as we noted in $\S 4.3$, these had not been accompanied by observations of velocity standard stars, and there were only three

observations. Without that data point, we would have no evidence for $K_{\mathrm{EB}}$ being larger than $\sim 10 \mathrm{~km} \mathrm{~s}^{-1}$, and a much lower remnant mass would be compatible with $f(M)$, even if the orbital period is significantly longer than $0.2 \mathrm{yr}$. Indeed, taking $K_{\mathrm{EB}}=10 \mathrm{~km} \mathrm{~s}^{-1}$ and holding the other terms in Equation (2) the same as above, gives $M_{3} \approx 0.73 M_{\odot}$. This estimate becomes $M_{3} \approx 0.85 M_{\odot}$ if $P_{3}=0.3 \mathrm{yr}$.

\section{Nebular Reddening}

A detailed study of the kinematics of the nebula has been carried out by Jones et al. (2009). However they did not measure the nebular reddening, which we wish to compare to the stellar reddening of NSV 19992. The aim here is to see how similar they are - very 
different values would be an argument for the PN and the binary being a chance line-of-sight alignment.

We used an observation from the AAT's RGO 25cm+TEK 250B grating, taken on 1995 January 7 . The spectra were taken at a PA of $0^{\circ}$ and with an exposure time of 1200 s. Arc spectra (Cu-Ar) were taken as well as wide-slit spectra of the flux-standard star $\mathrm{BD}+8^{\circ} 2015$. The standard reductions were followed, including a 2D wavelength calibration. The wavelength coverage was $3760-7394$ at $3.6 \AA$ pix $^{-1}$. Flux calibration of the wide-slit SuWt 2 spectral image was performed and transferred to the narrow-slit spectral image. Then a very narrow region of the 2D narrow-slit spectrum of SuWt 2 was extracted, close to the central star and thus within the nebular ring. Sky subtraction for this extracted spectrum could not be done from these observations because the telluric lines were contaminated by nebular emission. Instead we used a scaled spectrum of the telluric emission taken from an observation of another star on the same night. No extinction or airmass corrections were applied.

To calculate the nebular reddening, $c(\mathrm{H} \beta)$, we compared the measured value of $\mathrm{H} \alpha / \mathrm{H} \beta$ to the predicted ratio for nebular gas of $10000 \mathrm{~K}$ and $\log \left(n_{e}\right)=4$ (Hummer \& Storey 1987). Contamination of the Hi lines by He II for this nebula is less than a few percent and a similar percentage for $\mathrm{H} \alpha$ and $\mathrm{H} \beta$. We adopted the Galactic extinction law of Howarth (1983). The Balmer ratio, $5.18 \pm 0.78$, results in a value of $c(\mathrm{H} \beta)$ of $0.83 \pm 0.20$.

To compare this value to the stellar reddening we can use the conversion $E(B-V)=$ $c(\mathrm{H} \beta) / 1.492$, which is based on the Howarth reddening law, yielding $E(B-V)=0.56 \pm 0.14$. This is formally larger than the stellar value of $E(B-V)=0.40 \pm 0.05$, but consistent at just outside the $1 \sigma$ level. One should also bear in mind that when comparing the stellar and nebular values we make a number of approximations: (i) we assume that all the nebular reddening is interstellar; there will be some internal reddening although it is normally 
considered to be fairly low compared to the interstellar, (ii) the conversion between $c(\mathrm{H} \beta)$ and $E(B-V)$ can vary, as it depends on the value of $R$ (total-to-selective extinction) and the stellar energy distribution. As an example, Kaler \& Lutz (1985) calculated conversion constants of 1.43 to 1.68 ; the latter would bring the nebular $E(B-V)$ down to 0.49 .

\section{Light-Curve Solution}

The photometry and light curve of the eclipsing system provides information on the orbital inclination, relative radii, relative temperatures, and apparent magnitudes of the two stars and their reddening. In this section we explain the procedures used to determine final stellar and orbital parameters for NSV 19992 from our photometry.

We performed a simultaneous fit to the $B V I$ light curve data and the radial-velocity data, using modeling tools described in detail in several recent EB analyses (e.g., Stassun et al. 2004, 2006, 2007, 2008; Gómez Maqueo Chew et al. 2009).

Briefly, we used the most recent version of the EB light-curve modeling code of Wilson \& Devinney (1971, updated 2007), as implemented in the PHOEBE package of Prša \& Zwitter (2005). We adopted the ephemeris for the system derived in $\$ 3.3$ and held it fixed throughout the fitting procedure. We additionally held the orbital eccentricity fixed at zero; adopting the slight non-zero eccentricity found from fitting the AAT radial-velocity data alone, $e=0.026$ (see $\S 6.2$ ), changes the results presented here negligibly relative to the uncertainties.

In order to maintain control of the solution and its many free parameters, we performed this fitting in stages. Initially we held the orbital parameters fixed at the values determined from the double-lined spectroscopic orbit (\$함), and fixed the effective temperature of the primary component to the value determined from our spectral analysis $(9250 \mathrm{~K}$, $\$ 5$ ). This 
allowed us to determine an initial solution for the temperature of the secondary component and the sum of the component radii. Next we added the system inclination as a fitted parameter. We assumed a linear cosine limb-darkening law, with coefficients linearly interpolated from the tables of Van Hamme (1993). In addition we assumed an albedo of 1.0 and a gravity-brightening coefficient of 1.0 for both components, as appropriate for hot stars. We held the rotation rates of the two stars at their spectroscopically determined values of 17 and $<5 \mathrm{kms}^{-1}$, respectively. Finally, we performed a fit of all free parameters together, with the limb-darkening coefficients adjusted between iterations.

Due to the lack of a strong reflection or geometric effect in the light curves, and considering our assumed circular orbit, we are unable to constrain the individual component radii from the light curves alone, only their sum. Thus, in our light-curve fitting we solved for the sum of the radii, and then separately determined the ratio of the radii by requiring the ratios of the radii and the temperatures together to yield the correct luminosity ratio as determined from the spectra (see $\$ \underline{5}$ ).

The final light-curve fits are shown in Fig. 9 and the resulting component parameters are summarized in Table 9. We see that the eclipsing system consists of two nearly identical early A-type stars, both with masses near $2.7 M_{\odot}$, and nearly identical temperatures and radii which place them just above the main sequence.

The combined radius of the two stars calculated here is $8.2 R_{\odot}$ (Table 9), which compares fairly well to the value $5.4 R_{\odot}$ obtained from the angular diameter calculated in $\S 5.1$ (0.022 mas) and distance that will be calculated in $\S 9.2(2.3 \mathrm{kpc})$, combined with the small-angle approximation. The radiometric radius in $\S 5.1$ is of course very sensitive to the adopted effective temperature. 


\section{Discussion}

\subsection{Evolutionary Status of the Components of NSV 19992}

In Fig. 10 we compare the measured temperatures and radii of the two components of NSV 19992 with Yonsei-Yale ("Y2") evolutionary tracks (see Demarque et al. 2004 and references therein). The upper panel shows the early evolution of a single star of mass $2.69 M_{\odot}$ (the mean mass of the nearly identical components) and solar metallicity, starting from the zero-age MS (lower left corner), across the Hertzsprung gap, and to the base of the red-giant branch. The dotted curves represent the same evolutionary track but for metallicities of $[\mathrm{Fe} / \mathrm{H}] \pm 0.14$, representative of the $1 \sigma$ uncertainty in the measured Fe abundance of the system (see Table 4). The two overlapping filled circles show the effective temperatures and radii of the components of NSV 19992. Gratifyingly, both points lie precisely on the track corresponding to their dynamical masses.

Note that the very small errors on the temperatures represent the highly accurate temperature ratio determined from the light-curve solution; however, there is in addition a systematic error of $250 \mathrm{~K}$ on the absolute temperature scale (see Table 4), which is indicated in the panel. As the individual radii are connected by the accurately determined radius sum and ratio (see Table 9), their relative placement in the figure cannot be changed by more than the error bars shown on the individual points.

For visual simplicity, we have plotted the evolutionary tracks for only a single mass (that of the mean mass of the components); the difference in the positioning of the tracks for masses within the $1 \sigma$ mass uncertainties is less than that shown for the $1 \sigma$ range in $[\mathrm{Fe} / \mathrm{H}]$ (dotted tracks). Finally, for reference, absolute ages (in Myr) from the model are

shown at several points along the track. The implied ages of the two components are close to 520 Myr. 
Both components of NSV 19992 appear to occupy a remarkably short-lived phase of evolution. Even with the systematic uncertainty on the absolute temperatures of the components, it is clear that they reside in the "blue hook" that occurs just prior to the Hertzsprung gap, and which lasts in total only $\sim 1$ Myr. In spite of the brevity of this evolutionary phase, a small number of other EBs are also known in a similar state of evolution, including SZ Cen (Popper 1980), V1031 Ori (Andersen et al. 1990), and WX Cep (Popper 1987). As discussed by Andersen et al. (1990), it is surprising that so many systems have been seen in such a very short-lived state of evolution. One possible explanation is that the interior physics of the theoretical models (e.g., convective overshooting) could move the stars to be on the "red hook" instead of the "blue hook," the former being a somewhat longer-lived stage $(\sim 10$ Myr instead of $\sim 1 \mathrm{Myr})$.

The evolutionary rapidity in this portion of the H-R diagram can be used to further constrain the mass ratio of the components. By examining the range of stellar masses (at solar metallicity) for which the $\mathrm{Y}^{2}$ models predict temperatures and radii consistent with the measured values, we find that a minimum mass ratio of $q=0.9996$ (at $1 \sigma$ confidence) is required to place the two stars this closely in temperatures and radii.

We point out that these stellar evolutionary models represent the evolution of single stars only. The evolution through the blue-hook phase depends heavily on the details of convection and mixing which are still treated somewhat simplistically in the models. For binary systems, with an (as-yet) unknown mass-transfer history, the situation will be even more complex. However, a detailed examination of constraints on the model physics is beyond the scope of this observational paper. 


\subsection{Distance, Nebular Size and Age, and Source of Ionization}

Using the measured temperatures and radii for the components of NSV 19992 we can estimate the distance to the system. We find a bolometric absolute magnitude for the combined light of both stars of $M_{\mathrm{bol}}=-1.09$, which, together with a bolometric correction of $\mathrm{BC}_{V}=-0.07$, gives $M_{V}=-1.02$. The observed $V=12.00$ and $E(B-V)=0.40$ yield an unreddened $V_{0}=10.76$, from which we derive a distance $d=2.3 \pm 0.2 \mathrm{kpc}$ (including uncertainties in the stellar radii and temperatures, and in the reddening).

At this distance, the measured semimajor axis of the SuWt 2 nebula of $43^{\prime \prime}$ corresponds to a physical ring radius of 0.47 pc. Jones et al. (2009) have found a nebular expansion velocity of $28 \mathrm{~km} \mathrm{~s}^{-1}$, which when combined with the radius implies a dynamical age for the ring of $\sim 17,000 \mathrm{yr}$.

Interestingly, Smith et al. (2007) have speculated that the nebula may in fact be externally ionized by UV light from the angularly nearby 7th-mag B supergiant HD 121228 (the very bright star seen in Fig. 1), rather than from the central stellar system. HD 121228 has been classified B2 Ib by Humphreys (1975) and B1 II by Garrison et al. (1977). Of course, an early B supergiant could not be coeval with A-type stars having ages of $520 \mathrm{Myr}$, so the association would have to be one that occurred by chance. The $73^{\prime \prime}$ angular separation of HD 121228 and the central star of SuWt 2 corresponds to a projected physical separation of only 0.8 pc, if the two are at the same distance. The distance to HD 121228 has been estimated to be $2.8 \mathrm{kpc}$ by Westin (1985), based on Strömgren photometry. Although somewhat larger than the distance we derive for SuWt 2, it is probably not inconsistent, within the respective uncertainties. However, combined with the requirement for a chance approach to the nebula by a much younger star, and the fact that an external source of ionization still leaves the source of the nebular ejection unexplained, we remain doubtful of a physical association of the nebula and HD 121228. We prefer an interpretation 
in which the pair of A stars has a companion which both ejected and ionized the nebula, as described in more detail below.

\subsection{Non-synchronous Rotations of the Components}

With an orbital period of 4.9 days, the components of NSV 19992 might be expected to be rotating synchronously with the orbit. Indeed, all of the A-type binaries with orbital periods shorter than 3 days in the study of Matthews \& Mathieu (1993) were found to be circularized and synchronized, as well as most binaries with periods shorter than $\sim 10$ days. However, our study has demonstrated that, while the orbit of NSV 19992 is very likely circular, both components rotate much more slowly $\left(\sim 17\right.$ and $<5 \mathrm{kms}^{-1}$ for the primary and secondary, respectively) than the synchronous rotation speed of $\sim 43 \mathrm{~km} \mathrm{~s}^{-1}$.

The system is similar in this respect to V1031 Ori, a 3.4-day eclipsing system which is one of the very few close binaries in which the stars are known to rotate more slowly than synchronously (Andersen et al. 1990). A possible explanation is that the stars were once in synchronous rotation, but have slowed down through conservation of angular momentum as their radii rapidly expanded upon leaving the MS. However this by itself does not explain the dissimilar rotation rates of the two components in NSV 19992 - considering that they are of nearly identical mass and radius - unless the change in internal structure of these stars is an extremely strong function of mass and age.

We note that the primary ("fast") component of NSV 19992 has a comparable $v \sin i$ to what would be expected for synchronous rotation on the MS, when it had a radius of $\sim 1.7 R_{\odot}$. Thus we may speculate that the system may originally have been a wider, eccentric system, in which only the primary component was pseudo-synchronized (i.e., synchronized to its orbital angular velocity at periastron passage), and that the orbit only 
very recently became circularized when the NSV 19992 binary became embedded within the red-giant envelope of the tertiary companion.

In any event, NSV 19992 offers the potential for a detailed examination and stringent test of tidal evolution and CE evolution theory.

\subsection{The Case of the Missing UV Remnant}

As described in $\S 4.1$, at the time of our $I U E$ spectroscopy at the beginning of this project we were surprised by the lack of a detected hot companion in the binary. Here we re-examine whether this surprise was justified, in the light of what we have learned about the object.

In Fig. 11 we show the short-wavelength IUE spectrum of the SuWt 2 nucleus (from image SWP 41915), smoothed into 10- $\AA$ bins and plotted as a histogram. For comparison, we obtained from the IUE archive 7 the short-wavelength spectra of three nearby PNNi with well-determined distances: NGC 246 ( $d=495$ pc; Bond \& Ciardullo 1999), and NGC 6853 and NGC $7293(d=405$ and 216 pc, respectively; Benedict et al. 2009). The respective image numbers are SWP 42226, SWP 40066, and SWP 42066. We scaled these spectra to the 2300 pc distance and $E(B-V)=0.40$ reddening of NSV 19992, using the formulae of Cardelli, Clayton, \& Mathis (1989). These scaled and reddened spectra are also plotted in Fig. 11.

The figure shows that a relatively hot and luminous PNN like that of NGC 246 would have been detected easily if it were a companion of NSV 19992. However, the more evolved and fainter PNNi of NGC 6853 probably, and NGC 7293 certainly, would not have been

\footnotetext{
${ }^{7}$ available at http://archive.stsci.edu/iue/
} 
detected. Thus the lack of a detection of a central star in the UV here is not so unusual; the existing UV spectra do not rule out its presence.

\subsection{An Evolutionary Scenario}

The principal result of our study is that the optical central star of SuWt 2 consists of a close pair of A-type stars, both with masses close to $2.7 M_{\odot}$. Both of them have recently left the MS, and the system's age is about 520 Myr. Thus, neither star is a stellar remnant, and neither one could plausibly have ejected the surrounding PN, nor could either have supplied the UV flux required to ionize the nebula. A scenario in which one of the A stars is actually a stellar remnant in the process of a "born-again" thermal-pulse event also appears to be excluded.

We therefore need a third star, whose initial mass was greater than $2.7 M_{\odot}$, which has evolved to the AGB stage, ejected the PN, and is now an optically faint PNN or WD. Since we have presented evidence that the center-of-mass velocity of the central binary varies with time, we suggest that this unseen third body is in fact the remnant core of the star that ejected the nebula. The likely period of this third body is short enough that the binary could have been inside the envelope when it was an AGB star: a common envelope. The system, under our scenario, was thus a hierarchical triple. For the third star-the original primary - to have completed its evolution to the PN stage in $\sim 520 \mathrm{Myr}$, its initial mass need have been only slightly higher than the masses of the A-type pair, of order $\sim 2.9 M_{\odot}$. In the case of single-star evolution, such a star would produce a WD remnant of $\sim 0.7 M_{\odot}$ (e.g., Kalirai et al. 2008, Fig. 12), but the mass could be lower if the evolutionary growth of the core mass was truncated through a CE interaction.

Our picture is that the initial triple was wide enough for the primary to be able to 
evolve to the AGB stage, but then it engulfed the pair of A stars into a CE. Due to the high combined mass of the close A pair, the interaction must have been led quickly to a catastrophic ejection of the envelope, primarily into the orbital plane (e.g., as predicted in three-dimensional hydrodynamical CE simulations - for example Sandquist et al. 1998; Morris \& Podsiadlowski 2006; Nordhaus \& Blackman 2006), producing the very high density contrast between equator and poles that we observe in the ejected nebula. While most currently known post-CE PN central stars have short periods, of the order of hours to a few days, in this case the rapid ejection would have left the post-CE system still with a fairly long orbital period, as observed-assuming, of course, that the unseen third body in the SuWt 2 system is indeed the remnant core of the original primary

The orbital planes of the close EB and the more distant third body, in our scenario, need not be coplanar. Thus it is not necessarily surprising that the orbital inclination of the eclipsing pair, 81.5, differs from the viewing angle of the nebula of $\sim 68^{\circ}$.

To explain the lack of detection of the remnant in the IUE spectrum, we note that stellar remnants of $\sim 0.7 M_{\odot}$ cool and fade rapidly (e.g., Vassiliadis \& Wood 1994). Thus it is not implausible that the remnant could have faded to the UV brightness of the central stars of NGC 6853 or NGC 7293, as depicted above in Fig. 11, especially given the fairly advanced nebular age of $\sim 17,000 \mathrm{yr}$. In this picture, the PN was ionized earlier by UV radiation from the hot remnant, but is now beginning to recombine. A fast stellar wind from the hot remnant in its luminous stage would have inflated the faint bipolar lobes now seen lying perpendicular to the orbital plane.

In summary, our scenario suggests that the SuWt 2 system is the immediate product of a catastrophic event in which an orbiting combined mass of more than $5 M_{\odot}$ was ingested into the envelope of an AGB star, leading to ejection of the envelope into the orbital plane. The distant future of this system may be destined to be even more catastrophic. As the A 
stars evolve, they may merge into a single star, which will then expand to become a red giant or AGB star, engulfing the third body into a second common envelope, which will likewise be ejected. The product may be a close pair of white dwarfs whose combined mass exceeds the Chandrasekhar limit, so that ultimately the system may perish as a Type Ia supernova.

\subsection{Future Work}

The most obvious two goals of future work on NSV 19992/SuWt 2 are:

- To measure the variations of the systemic velocity with time, to confirm its variability and better constrain the mass of the possible third body in the system. Unfortunately, the reflex motion of the eclipsing pair for the unseen masses discussed above is only of order 1 light-minute, which is smaller than the typical $\sim 3$ min uncertainties in our eclipse timings, so we cannot verify the presence of a third body from our timing data. Radial-velocity monitoring will require much patience because of the almost 5-day orbit and the lack of many facilities for long-term spectral monitoring.

- To obtain UV data to directly detect a third, hot body. As we have pointed out, the lack of a detection of a central star of SuWt 2 in the existing UV from IUE does not provide a strong constraint. Unfortunately, the low-latitude field surrounding SuWt 2 cannot be observed by GALEX, according to the brightness-checking tool 8 However, deeper UV spectroscopy than has been obtained with IUE, e.g., with the HST, might be capable of revealing the hot companion.

\footnotetext{
${ }^{8}$ http://sherpa.caltech.edu/gips/tools/chkbstar.html
} 
H.E.B. thanks Michael Meakes for making the IUE observations described here, Robin Ciardullo and Noam Soker for discussions, and numerous telescope operators, support personnel, and service observers at CTIO, and the SMARTS telescope schedulers at Yale and Stony Brook Universities. He also thanks the STScI Director's Discretionary Research Fund for partial support of this work. We thank Tom Marsh for the use of MOLLY and PAMELA. We thank Tanmoy Laskar for his provision of standard-star theoretical spectra, and Kenneth De Smedt-Van Gent and Joris Vos for their thorough analysis of the standard-star radial velocities. K.G.S. acknowledges the support of NSF Career grant AST-0349075, NSF AST-0849736, a Cottrell Scholar award from the Research Corporation, and the hospitality of the Space Telescope Science Institute's Caroline Herschel Distinguished Visiting Scholars program. Some of the data presented in this paper were obtained from the Multimission Archive at the Space Telescope Science Institute (MAST). STScI is operated by the Association of Universities for Research in Astronomy, Inc., under NASA contract NAS5-26555. Support for MAST for non-HST data is provided by the NASA Office of Space Science via grant NAG5-7584 and by other grants and contracts. This research has made use of the SIMBAD database, operated at CDS, Strasbourg, France. This research used the facilities of the Canadian Astronomy Data Centre operated by the National Research Council of Canada with the support of the Canadian Space Agency. This paper uses observations made at the South African Astronomical Observatory (SAAO).

Facilities: SMARTS:1.5m, SMARTS:1.3m, CTIO:0.9m, CTIO:1.5m, IUE, SAAO, AAO, ESO 


\section{REFERENCES}

Andersen, J., Nordstrom, B., \& Clausen, J. V. 1990, A\&A, 228, 365

Andrews, A. D., \& Lindsay, E. M. 1967, Irish Astronomical Journal, 8, 126

Balick, B., \& Frank A., 2002, ARA\&A, 40, 439

Benedict, G. F., et al. 2009, AJ, 138, 1969

Blackwell, D. E., \& Shallis, M. J. 1977, MNRAS, 180, 177

Bond, H. E. 2000, in Asymmetrical Planetary Nebulae II: From Origins to Microstructures, 199,115

Bond, H. E., \& Ciardullo, R. 1999, PASP, 111, 217

Bond, H. E., Ciardullo, R., \& Webbink, R. 1996, Bulletin of the American Astronomical Society, 28, 1401

Bond, H. E., Exter, K., \& Pollacco, D. L. 2001, Bulletin of the American Astronomical Society, 33, 1510

Bond, H. E., Exter, K., Smalley, B., Maxted, P., Pollacco, D., \& Stassun, K. 2008, Bulletin of the American Astronomical Society, 40, 530 
Bond, H. E., \& Livio, M. 1990, ApJ, 355, 568

Bond, H. E., O’Brien, M. S., Sion, E. M., Mullan, D. J., Exter, K., Pollacco, D. L., \& Webbink, R. F. 2002, Exotic Stars as Challenges to Evolution, 279, 239

Bond, H. E., Pollacco, D. L., \& Webbink, R. F. 2003, AJ, 125, 260

Cardelli, J. A., Clayton, G. C., \& Mathis, J. S. 1989, ApJ, 345, 245

De Marco, O. 2009, PASP, 121, 316

De Marco, O., Bond, H. E., Harmer, D., \& Fleming, A. J. 2004, ApJ, 602, L93

Demarque, P., Woo, J.-H., Kim, Y.-C., \& Yi, S. K. 2004, ApJS, 155, 667

Dobrinčić, M., Villaver, E., Guerrero, M. A., \& Manchado, A. 2008, AJ, 135, 2199

Exter, K., Bond, H., Pollacco, D., \& Dufton, P. 2003, Planetary Nebulae: Their Evolution and Role in the Universe, 209, 234

Garrison, R. F., Hiltner, W. A., \& Schild, R. E. 1977, ApJS, 35, 111

Gómez Maqueo Chew, Y., Stassun, K. G., Prša, A., \& Mathieu, R. D. 2009, ApJ, 699, 1196

Gudehus, D. H. 2001, Bulletin of the American Astronomical Society, 33, 850 
Howarth, I. D. 1983, MNRAS, 203, 301

Hummer, D. G., \& Storey, P. J. 1987, MNRAS, 224, 810

Humphreys, R. M. 1975, A\&AS, 19, 243

Hynes, R. I., \& Maxted, P. F. L. 1998, A\&A, 331, 167

Jacoby, G., et al. 2010, Bulletin of the American Astronomical Society, 41, 472

Jones, D., Lloyd, M., Mitchell, D. L., Pollacco, D. L., O’Brien, T. J., \& Vaytet, N. M. H. 2009, MNRAS, 401, 405

Kaler, J. B., \& Lutz, J. H. 1985, PASP, 97, 700

Kalirai, J. S., Hansen, B. M. S., Kelson, D. D., Reitzel, D. B., Rich, R. M., \& Richer, H. B. 2008, ApJ, 676, 594

Kazarovets, E. V., Samus, N. N., \& Durlevich, O. V. 1998, Information Bulletin on Variable Stars, 4655,1

Kurucz, R. L. 1993, Kurucz CD-ROM 13: ATLAS9, SAO, Cambridge, USA

Kwok, S. 2008, The Art of Modeling Stars in the 21st Century, Proceedings of the International Astronomical Union, IAU Symposium, Volume 252, pp 197-203 
Lafler, J., \& Kinman, T. D. 1965, ApJS, 11, 216

Landolt, A. U. 1992, AJ, 104, 340

Matthews, L. D., \& Mathieu, R. D. 1993, in ASP Conf. Series, Massive Stars: Their Lives in the Interstellar Medium, eds J. P. Cassinelli, E. B. Churchwell, 35, 223

Miszalski, B., Acker, A., Moffat, A. F. J., Parker, Q. A., \& Udalski, A. 2008a, A\&A, 488, L79

Miszalski, B., Parker, Q. A., Acker, A., Birkby, J. L., Frew, D. J., \& Kovacevic, A. 2008b, MNRAS, 384, 525

Miszalski, B., Acker, A., Moffat, A. F. J., Parker, Q. A., \& Udalski, A. 2009, A\&A, 496, 813

Morris, T., \& Podsiadlowski, P. 2006, MNRAS, 365, 2

Nordhaus, J., \& Blackman, E. G. 2006, MNRAS, 370, 2004

Parker, Q. A., et al. 2005, MNRAS, 362, 689

Parker, Q. A., et al. 2006, MNRAS, 373, 79

Popper, D. M. 1980, ARA\&A, 18, 115

Popper, D. M. 1987, AJ, 93, 672 
Przybilla, N., Butler, K., Becker, S. R., \& Kudritzki, R. P. 2006, A\&A, 445, 1099

Prša, A., \& Zwitter, T. 2005, ApJ, 628, 426

Sandquist, E. L., Taam, R. E., Chen, X., Bodenheimer, P., \& Burkert, A. 1998, ApJ, 500, 909

Schuster, H.-E., \& West, R. M. 1976, A\&A, 46, 139

Schwarz, H. E., Corradi, R. L. M., \& Melnick, J. 1992, A\&AS, 96, 23

Smalley, B., Smith, K. C., \& Dworetsky, M. M., 2001, UCLSYN Userguide

Smith, K. C. 1992, PhD thesis, University of London

Smith, N., Bally, J., \& Walawender, J. 2007, AJ, 134, 846

Stassun, K. G., Mathieu, R. D., Cargile, P. A., Aarnio, A. N., Stempels, E., \& Geller, A. 2008, Nature, 453, 1079

Stassun, K. G., Mathieu, R. D., \& Valenti, J. A. 2006, Nature, 440, 311

Stassun, K. G., Mathieu, R. D., \& Valenti, J. A. 2007, ApJ, 664, 1154

Stassun, K. G., Mathieu, R. D., Vaz, L. P. R., Stroud, N., \& Vrba, F. J. 2004, ApJS, 151, 357 
Stetson, P. B. 1987, PASP, 99, 191

Stetson, P. 2000, User's Manual for DAOPHOT II (Victoria: Dominion Astrophysical Observatory)

Tylenda, R., Siódmiak, N., Górny, S. K., Corradi, R. L. M., \& Schwarz, H. E. 2003, A\&A, 405,627

Van Hamme, W. 1993, AJ, 106, 2096

Unsöld, A. 1955, Physik der Sternatmosphären. Springer-Verlag, Berlin-GöttingenHeidelberg

Vassiliadis, E., \& Wood, P. R. 1994, ApJS, 92, 125

West, R. M. 1976, PASP, 88, 896

West, R. M., \& Schuster, H.-E. 1980, A\&A, 88, 350

Westin, T. N. G. 1985, A\&AS, 60, 99

Wilson, R. E., \& Devinney, E. J. 1971, ApJ, 166, 605

Zacharias, N., Monet, D. G., Levine, S. E., Urban, S. E., Gaume, R., \& Wycoff, G. L., 2004, BAAS, 205.4815 
This manuscript was prepared with the AAS LATEX macros v5.2. 


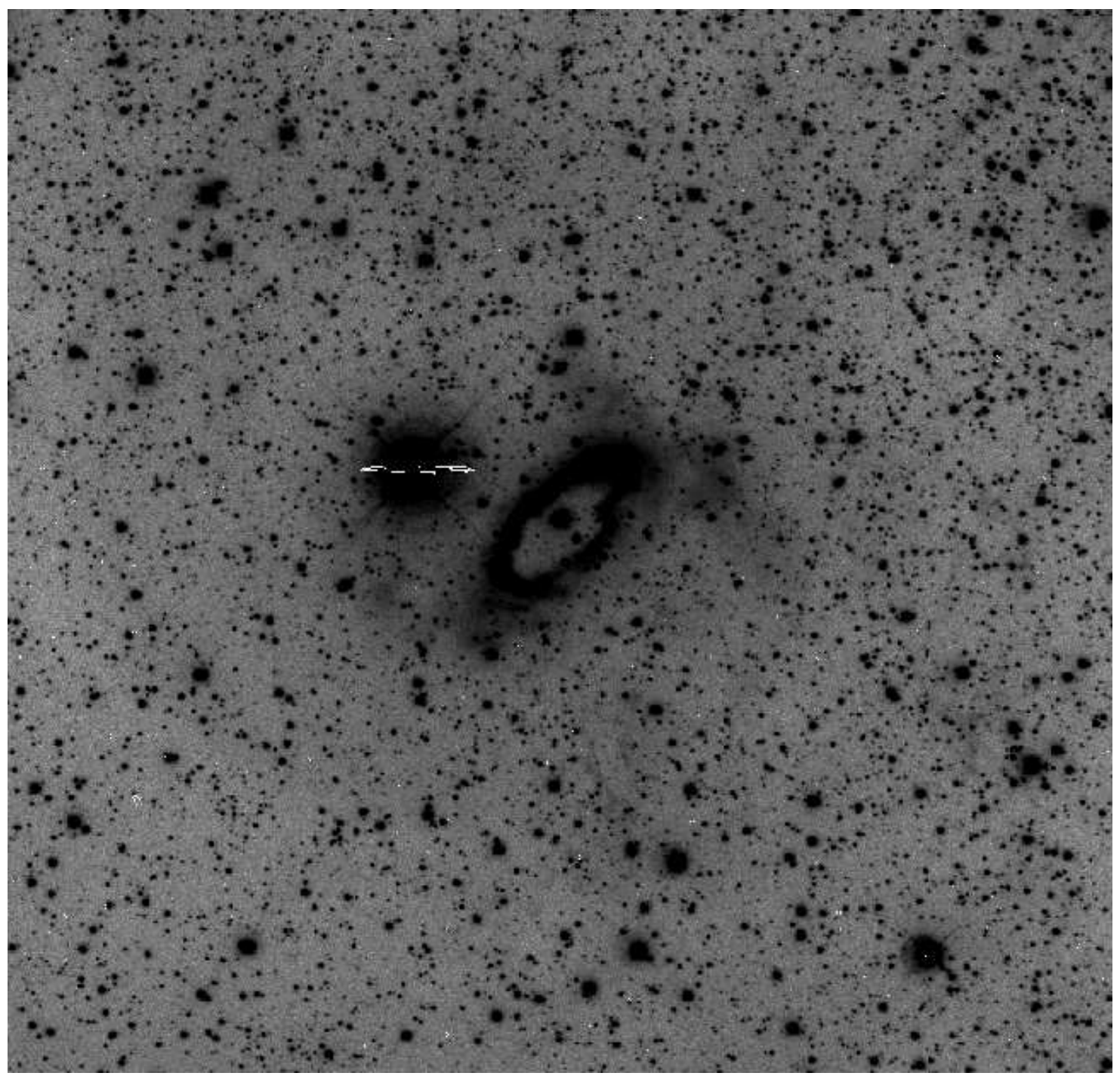

Fig. 1.- CCD image of SuWt 2, obtained with the CTIO 1.5-m telescope using an $\mathrm{H} \alpha+[\mathrm{N}$ II] filter and an exposure of $3 \times 600 \mathrm{~s}$. A logarithmic stretch has been used to illustrate the faint lobes extending perpendicularly to the bright nebular ring. $\mathrm{N}$ is up and $\mathrm{E}$ is left, and the image is $8^{\prime}$ high. The bright B-type supergiant HD 121228 lies $73^{\prime \prime}$ ENE of the central star. 


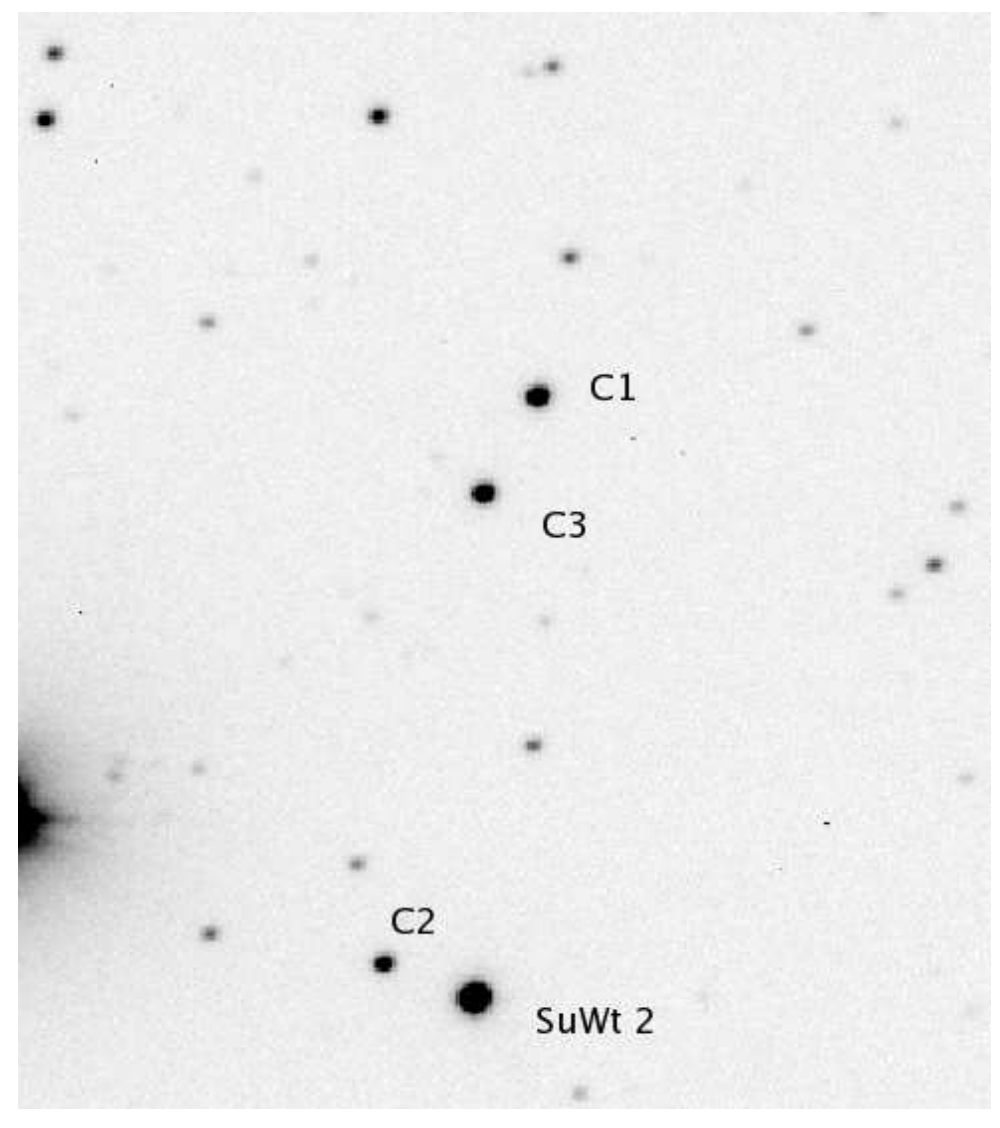

Fig. 2.- Finding chart for central star of SuWt 2 and its comparison stars, from a $B$-band frame obtained with the CTIO 0.9-m telescope. $\mathrm{N}$ is up and $\mathrm{E}$ is left, and the image is 2.7 high. 


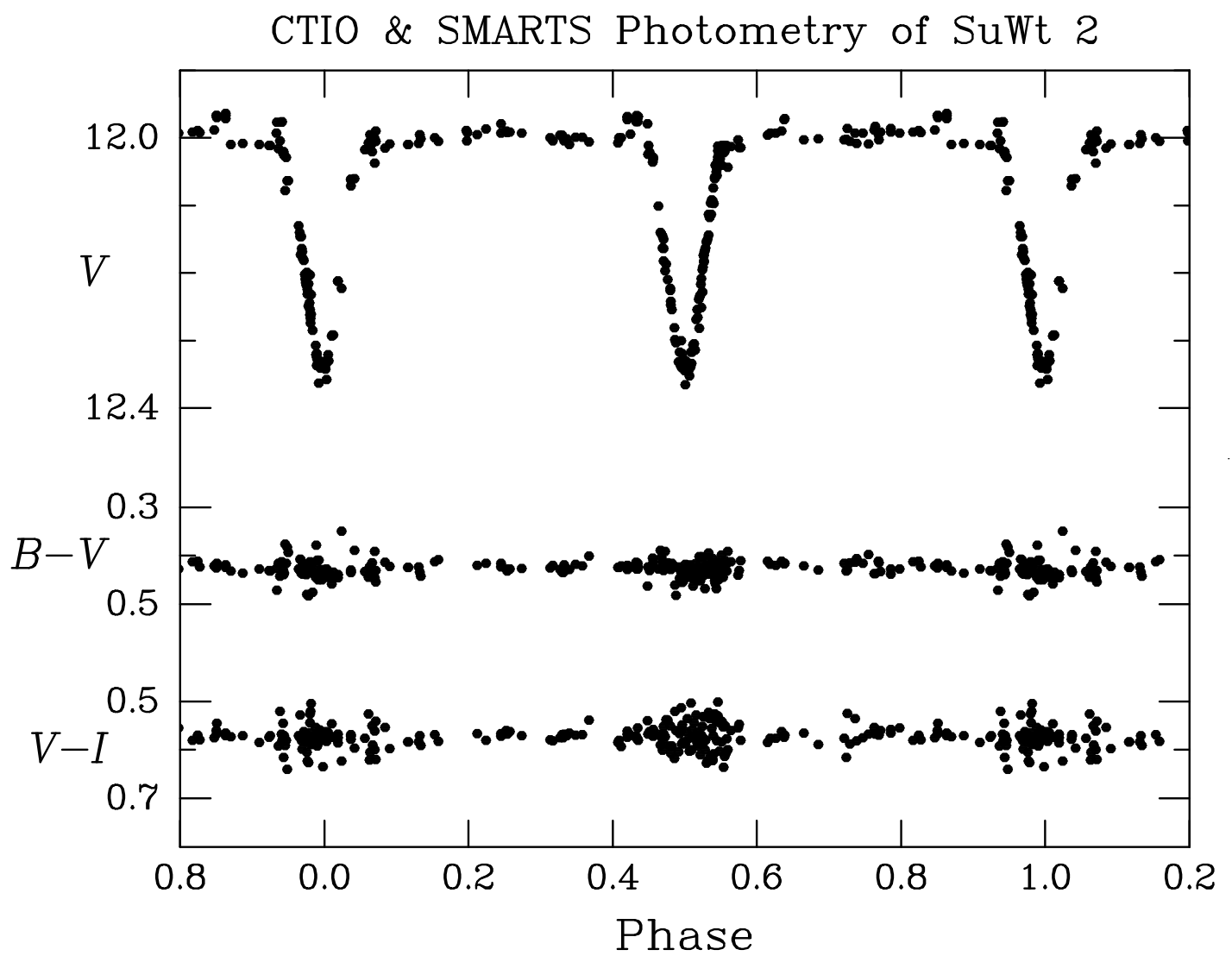

Fig. 3.- $V$-band light curve, and $B-V$ and $V-I$ color curves, of NSV 19992 phased with a period of 4.9098505 days. 


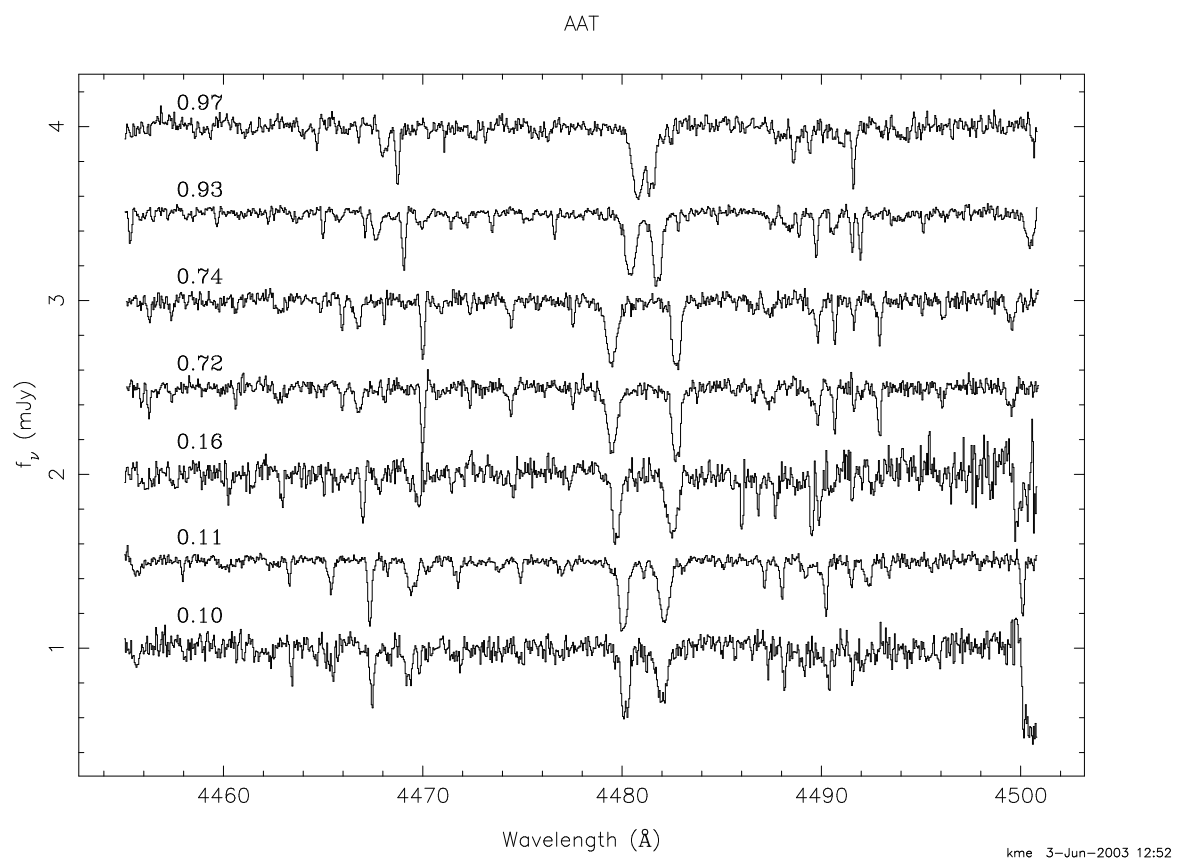

Fig. 4. - Spectra of NSV 19992 taken with the AAT echelle (the phases indicated are based on a preliminary ephemeris). The spectra have been continuum normalized and shifted successively upwards by 0.5. Flux is in arbitrary units. They clearly show the two stars moving in a binary orbit. 


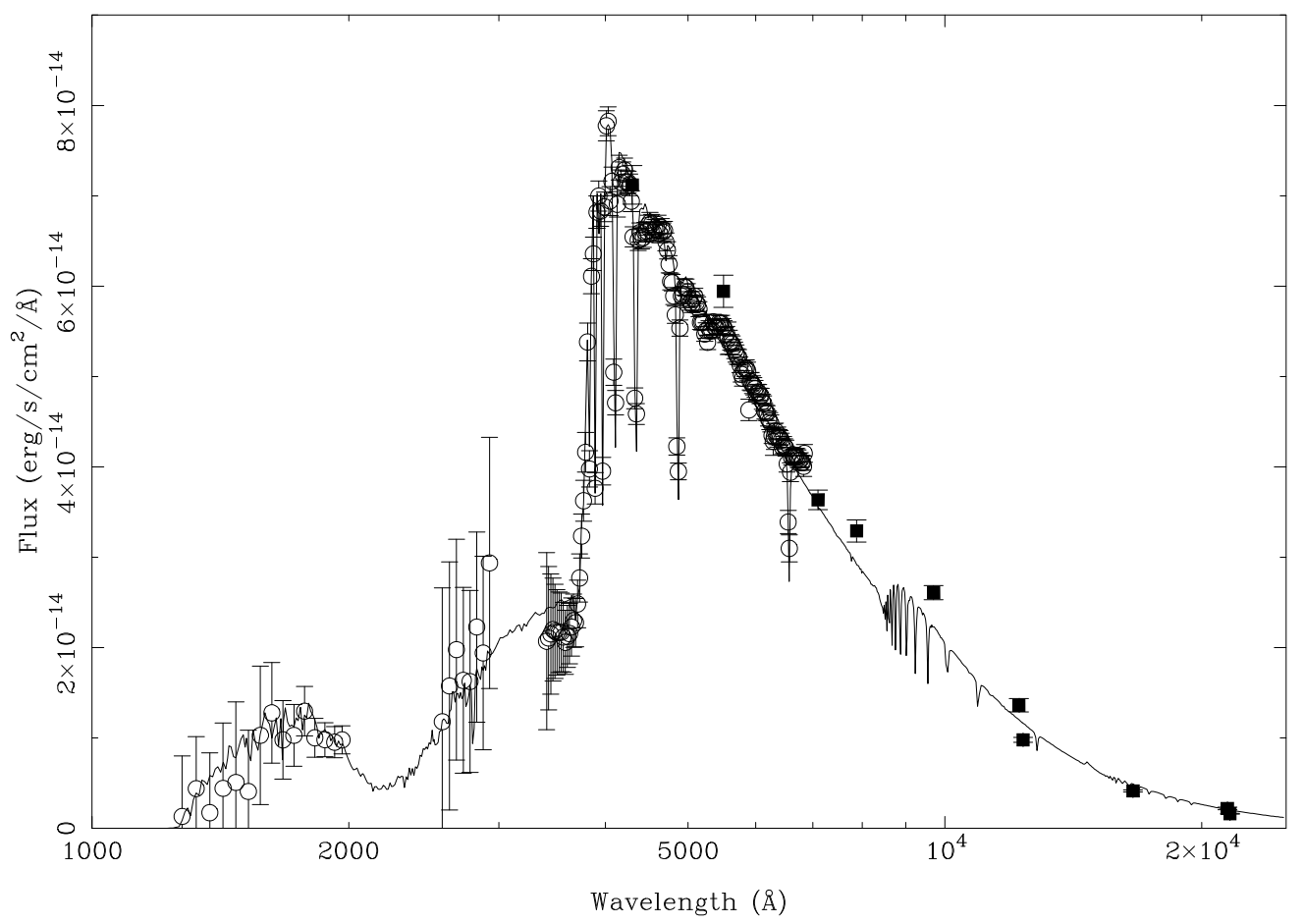

Fig. 5.- The spectral energy distribution, using spectra and photometry from various sources. Symbols: open-binned IUE and the low-resolution optical spectrum; filled squaresbroad-band photometry (optical, DENIS, 2MASS); continuous line-best fitting Kurucz model atmosphere flux distribution. $E(B-V)=0.40$. 

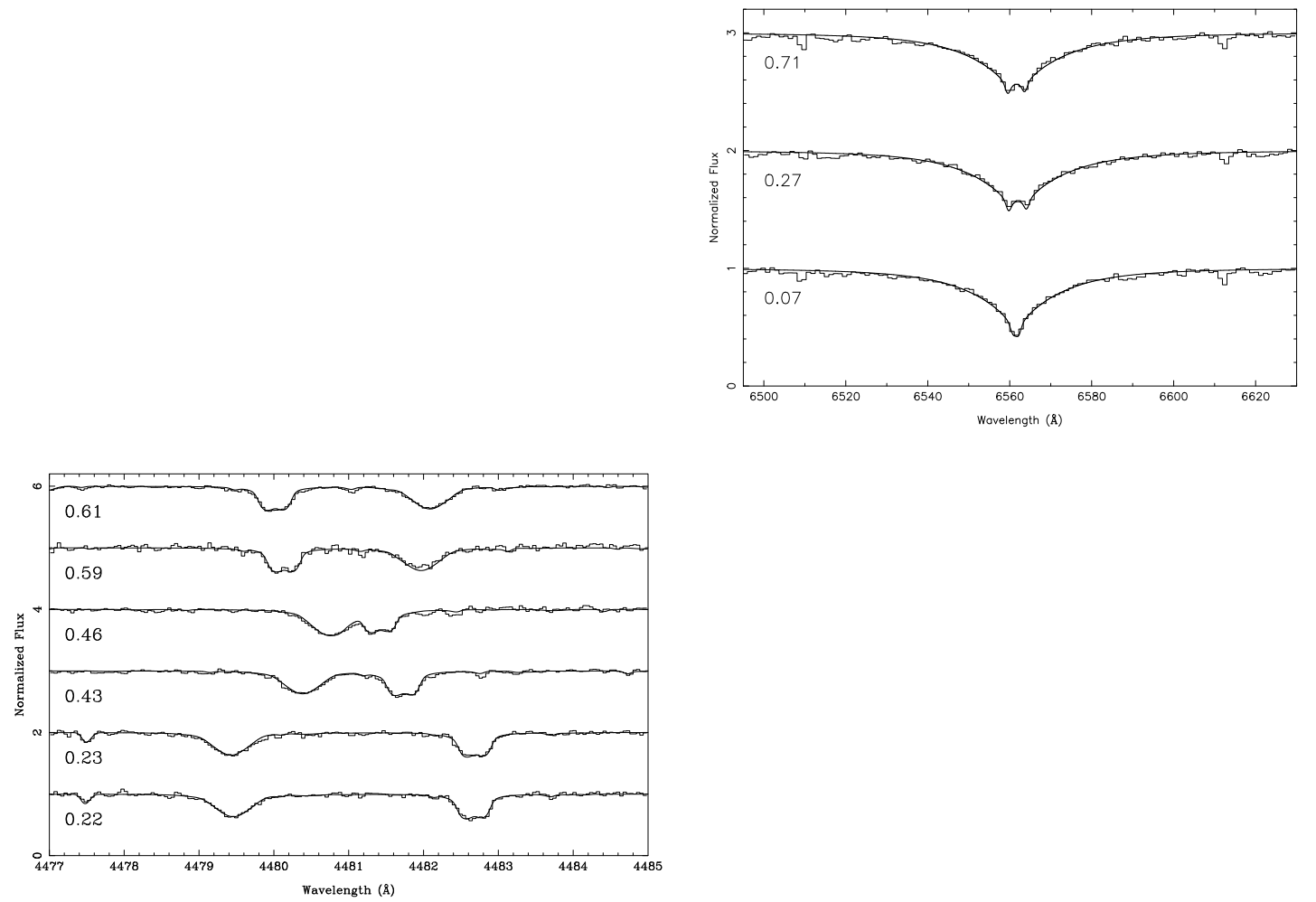

Fig. 6. - The echelle spectra and model fits to the Mg II $4481 \AA$ region (left) and H $\alpha$ (right). Histograms are the data and continuous lines are the synthetic profiles. These profiles have been fitted using the parameters determined by us; only the radial-velocity values have been allowed to vary between phases. Phases are marked. 

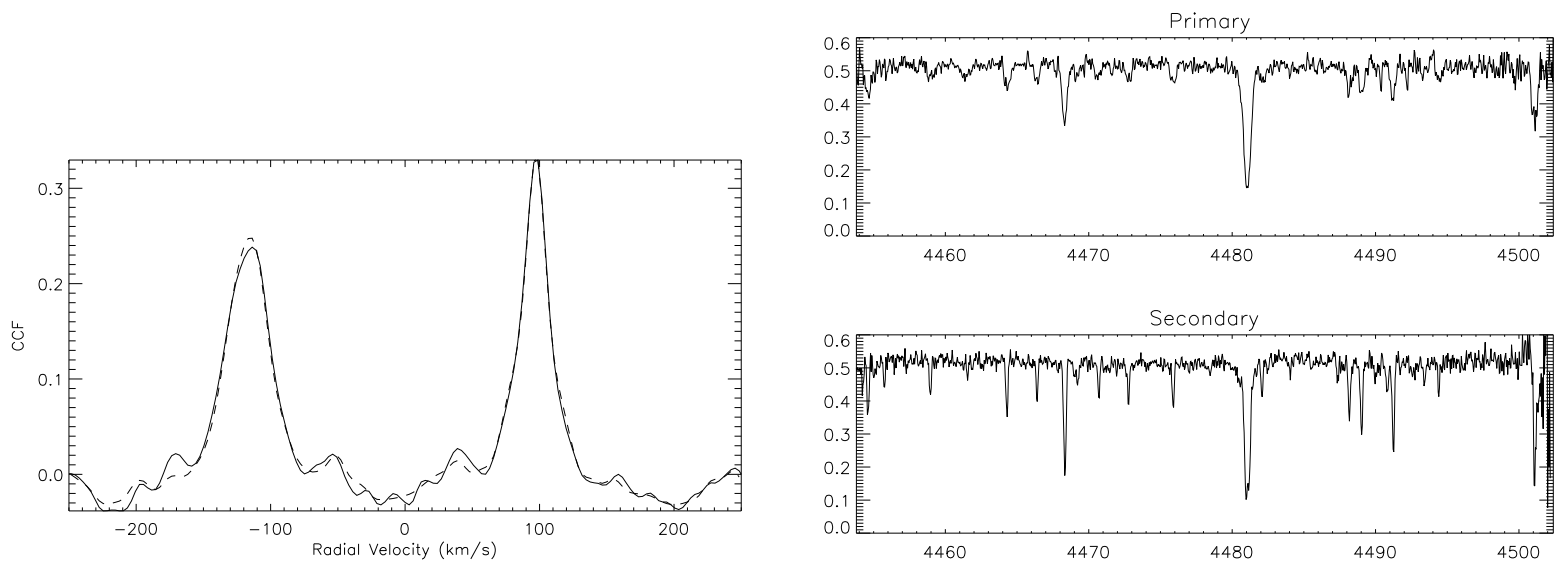

Fig. 7.- Left An example of fits to the peaks in the CCFs measured from one of the model fits to an AAT spectrum. Right Disentangled AAT echelle spectra showing the region around the Mg II line(s). Here one can see the two stars separately, clearly showing the great similarity in the spectra except for their line widths. 


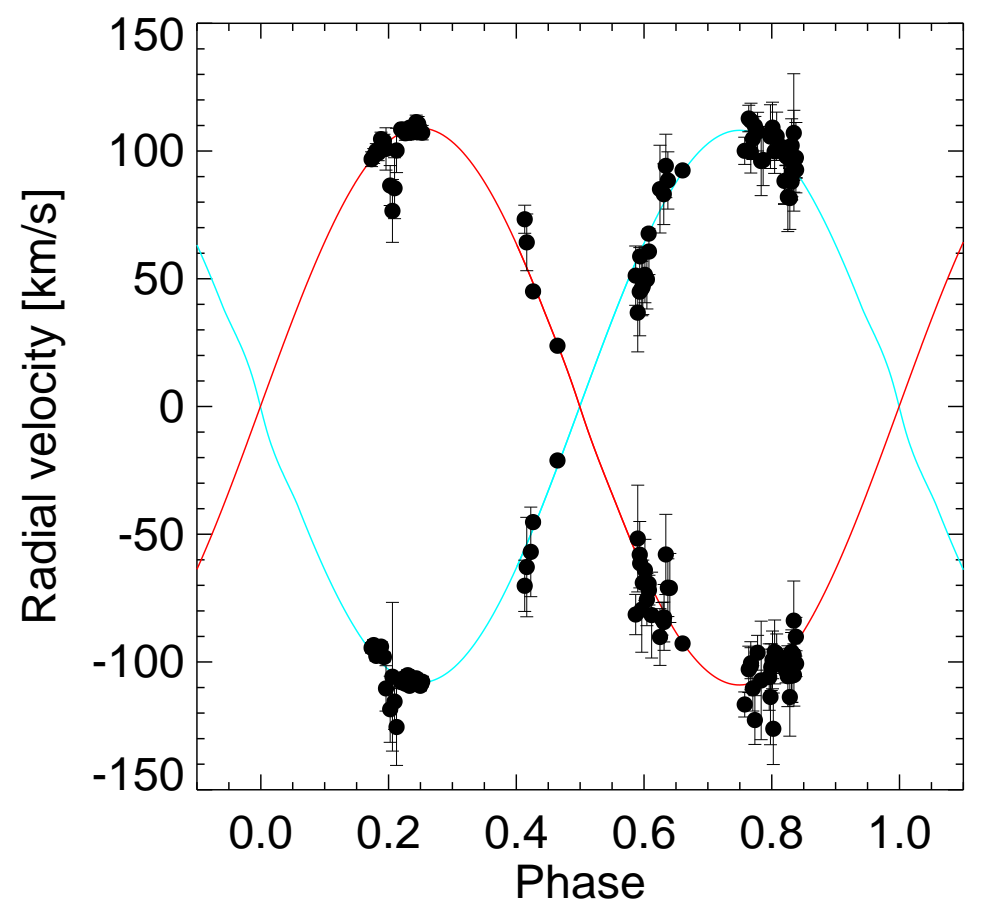

Fig. 8.- Orbital fit to the radial-velocity measurements of NSV 19992. Data from the different observing runs have been shifted by the systemic velocities listed in Table 7. Parameters of the orbit solution are summarized in Tables 8 and 9. The velocity curve of the primary component is shown in blue, and that of the secondary in red. 


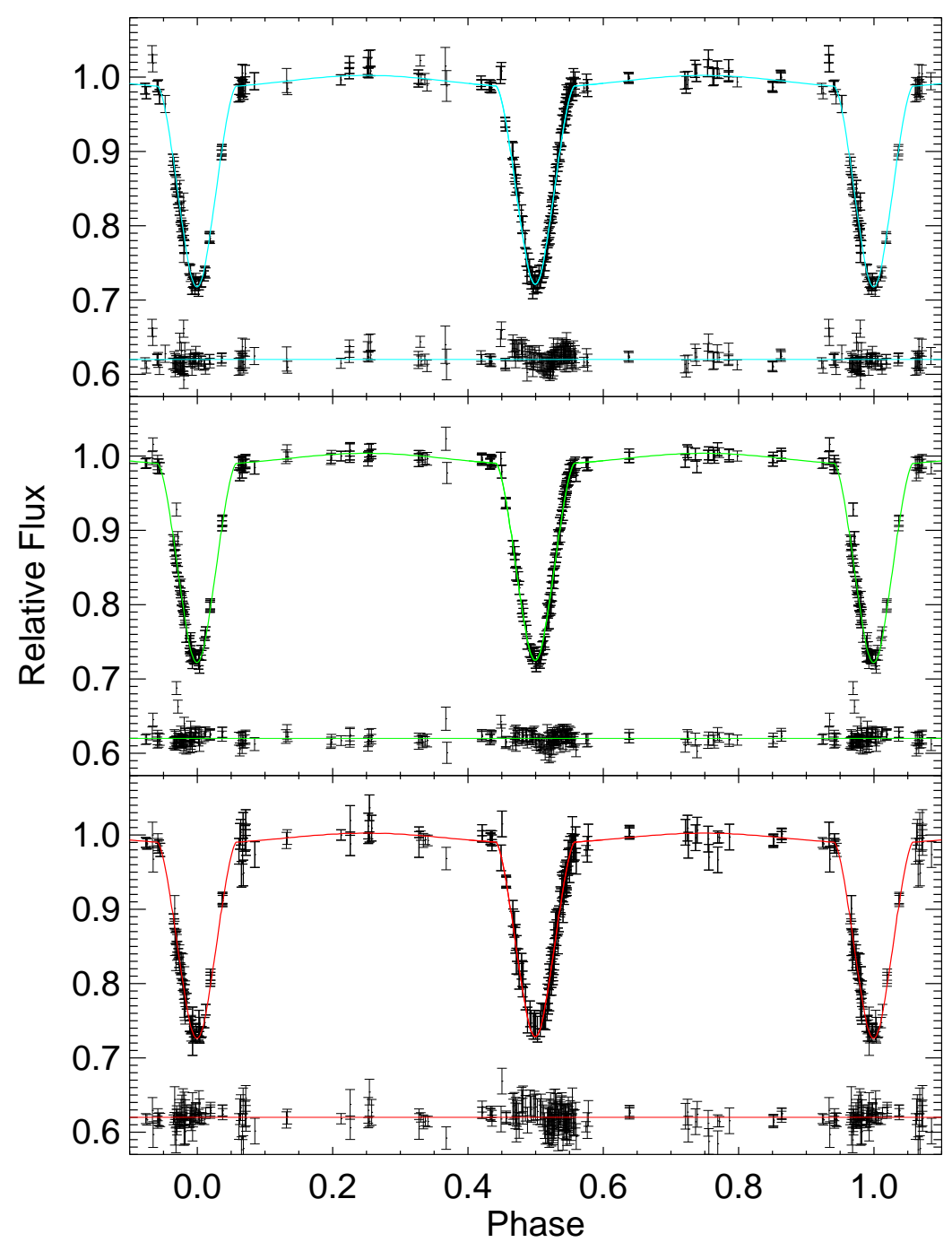

Fig. 9. - Light-curve fits for NSV 19992. The three panels show the light-curve data and fit for $B, V$, and $I$ bands, respectively. The final physical parameters of the NSV 19992 system resulting from this fit are summarized in Table 9. 

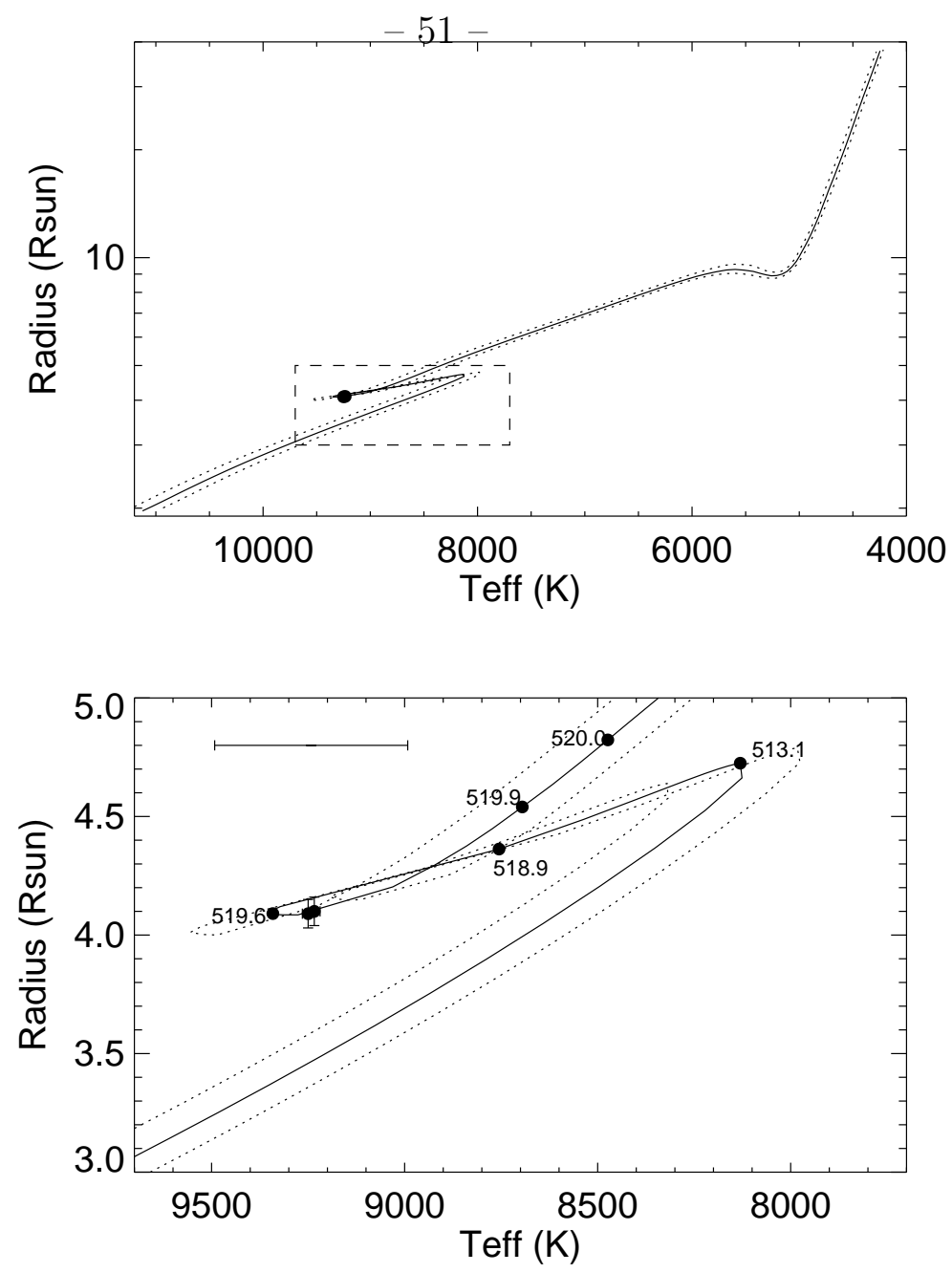

Fig. 10.- Evolutionary state of the components of NSV 19992 is shown in a modified H$\mathrm{R}$ diagram ( $T_{\text {eff }}$ vs. $R$ ). The solid line is an evolutionary track from the "Yonsei-Yale" theoretical models for a single star with mass $2.69 M_{\odot}$ and solar metallicity. The dotted curves represent the same model but for metallicity values of $[\mathrm{Fe} / \mathrm{H}] \pm 0.14$, representative of the $1 \sigma$ uncertainty in the measured Fe abundance. The measured values for the components of NSV 19992 are shown as filled symbols with error bars. The upper panel shows an overview of the evolution from the main sequence (lower left corner) to the base of the red-giant branch. The lower panel is an expanded view of the region around the red and blue "hooks" (indicated by the dashed box in the upper panel) representing the transition from core H-burning to shell H-burning. Stellar ages in Myr from the theoretical model are indicated at various points along the track. The bar near the top of the lower panel represents the systematic uncertainty on the stellar $T_{\text {eff }}$. 


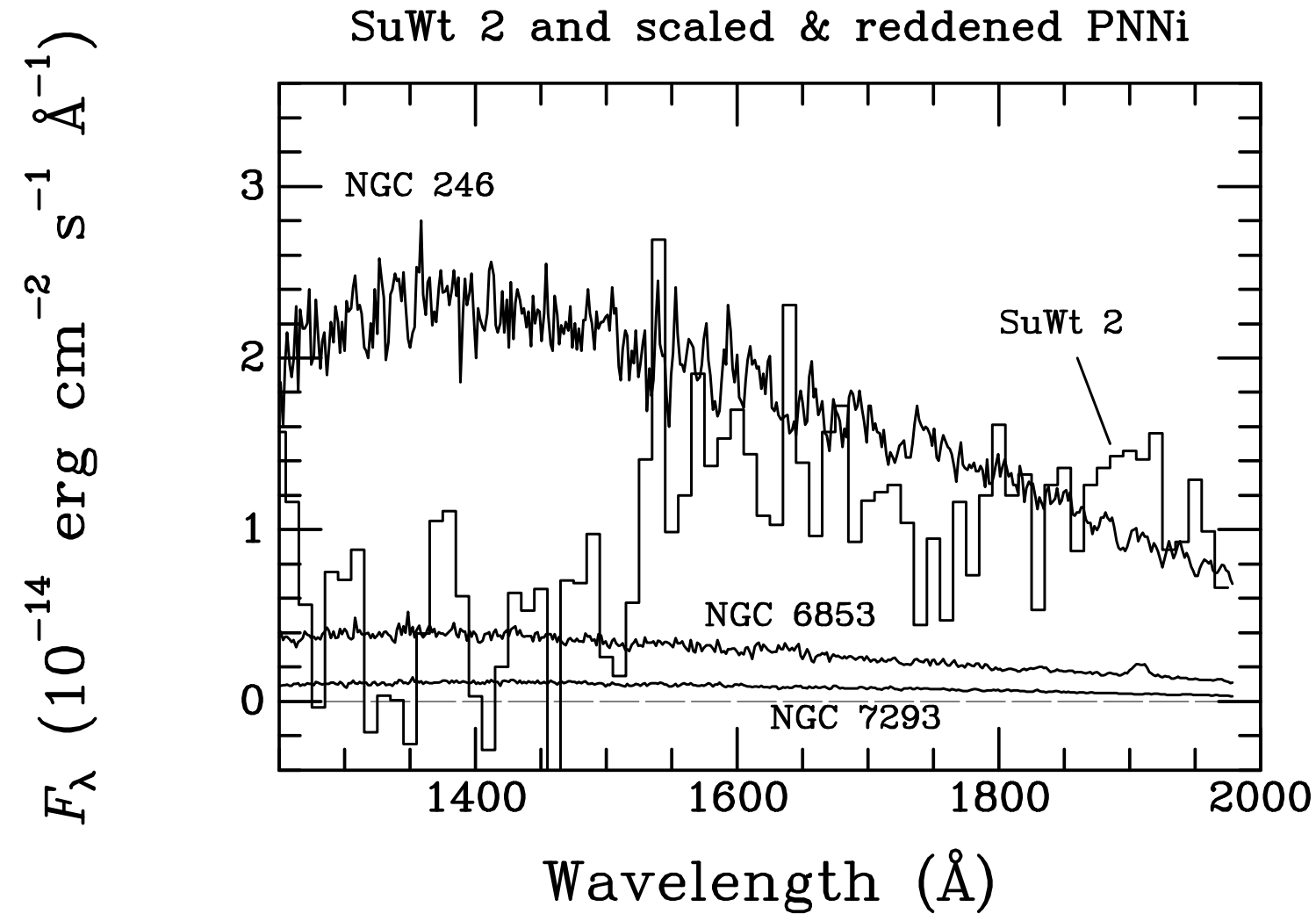

Fig. 11. - Short-wavelength IUE spectra of NSV 19992 (histogram; data smoothed to $10 \AA$ bins), NGC 246, NGC 6853, and NGC 7293 (continuous lines). The spectra of the last three have been scaled from their known distances to $2.3 \mathrm{kpc}$, and have also been reddened to a value of $E(B-V)=0.40$. The spectrum of NSV 19992 is consistent with that of a pair of early A-type stars. Although a PNN as hot and luminous as that of NGC 246 would have been detected if it were a companion of the binary, the other two would not have been detected conclusively at the $\mathrm{S} / \mathrm{N}$ of the spectrum. 
Table 1. Positions and calibrated photometry for NSV 19992 outside eclipse and the comparison stars. Also the DENIS and 2MASS magnitudes of NSV 19992

\begin{tabular}{lccccc}
\hline \hline \multicolumn{1}{c}{ Star } & $\alpha(\mathrm{J} 2000)$ & $\delta(\mathrm{J} 2000)$ & $V$ & $B-V$ & $V-I$ \\
\hline \multirow{2}{*}{ NSV 19992 } & 135543.23 & -592239.8 & $11.999 \pm 0.006$ & $0.424 \pm 0.002$ & $0.563 \pm 0.003$ \\
C1 & 135542.35 & -592115.2 & $12.826 \pm 0.006$ & $1.377 \pm 0.004$ & $1.440 \pm 0.004$ \\
C2 & 135544.89 & -592235.3 & $13.645 \pm 0.004$ & $1.285 \pm 0.004$ & $1.408 \pm 0.004$ \\
C3 & 135543.31 & -592129.0 & $13.742 \pm 0.004$ & $0.538 \pm 0.003$ & $0.689 \pm 0.003$ \\
& & & & & \\
NSV 19992 & DENIS mags & $\mathrm{I}=11.405 \pm 0.04$ & $\mathrm{~J}=10.918 \pm 0.06$ & $\mathrm{~K}=10.745 \pm 0.07$ \\
NSV 19992 & 2MASS mags & $\mathrm{J}=11.263 \pm 0.024$ & $\mathrm{H}=11.096 \pm 0.026$ & $\mathrm{~K}=11.063 \pm 0.025$ \\
\hline
\end{tabular}

Table 2. Eclipse timings of NSV 19992

\begin{tabular}{lccc}
\hline \hline HJD $(2450000+)$ & $E$ & $(O-C)[$ phase $]$ & Filter \\
\hline $599.8417 \pm 0.0013$ & -14 & -0.0024 & $B$ \\
$599.8475 \pm 0.0011$ & -14 & -0.0012 & $V$ \\
$599.8479 \pm 0.0012$ & -14 & -0.0012 & $I$ \\
$668.5857 \pm 0.0018$ & 0 & -0.0012 & $V$ \\
$668.5868 \pm 0.0027$ & 0 & -0.0010 & $I$ \\
$668.5907 \pm 0.0017$ & 0 & -0.0002 & $B$ \\
$923.9079 \pm 0.0029$ & 52 & +0.0008 & $B$ \\
$923.9085 \pm 0.0018$ & 52 & +0.0010 & $V$ \\
$1051.5509 \pm 0.0040$ & 78 & -0.0018 & $B$ \\
$1051.5611 \pm 0.0068$ & 78 & +0.0003 & $I$ \\
$1051.5648 \pm 0.0029$ & 78 & +0.0010 & $V$ \\
$1343.6958 \pm 0.0028$ & 137.5 & -0.0000 & $B$ \\
$1343.6959 \pm 0.0023$ & 137.5 & -0.0000 & $V$ \\
$1343.6974 \pm 0.0059$ & 137.5 & +0.0003 & $I$ \\
$1991.8001 \pm 0.0012$ & 269.5 & +0.0008 & $V$ \\
$1991.8016 \pm 0.0026$ & 269.5 & +0.0011 & $I$ \\
$1991.8029 \pm 0.0015$ & 269.5 & +0.0014 & $B$ \\
$4208.5838 \pm 0.0029$ & 721 & -0.0020 & $I$ \\
$4208.5861 \pm 0.0023$ & 721 & -0.0016 & $V$ \\
$4208.5925 \pm 0.0021$ & 721 & -0.0003 & $B$ \\
\hline & & & \\
\hline
\end{tabular}


Table 3. Details of the spectroscopic observing runs

\begin{tabular}{|c|c|c|c|}
\hline Date & Telescope & Wavelength Calbration & Exposure \\
\hline month/day[range]/year & & Range/Dispersion/RMS & \\
\hline $06 / 13 / 90$ & $I U E$ & 1150-1970 А; low & $4200 \mathrm{~s}$ \\
\hline $06 / 25 / 91$ & $I U E$ & 1150-1970 ̊; low & $13500 \mathrm{~s}$ \\
\hline $06 / 25 / 91$ & $I U E$ & 1850-3350 ̊;low & $1500 \mathrm{~s}$ \\
\hline $05 / 23-29 / 95$ & SAAO $1.9 \mathrm{~m}$ (RPCS) & $4080-5160 \AA ; 0.8 \AA ; 0.05-0.1 \AA \mathrm{pix}^{-1}$ & $1200 \mathrm{~s}$ \\
\hline $05 / 21-23 / 00$ & AAO AAT (UCLES) & 4455-4500 $\AA ; 0.12 \AA ;-{ }^{\mathrm{a}}$ & 1800 s \\
\hline $04 / 3-5 / 01$ & AAO AAT (RGO) & $4138-4577 \AA ; 0.55 \AA ; 0.02-0.03 \AA \mathrm{pix}^{-1}$ & $1200 \mathrm{~s}$ \\
\hline 04/19-22/95 & ESO NTT (EMMI) & $4080-5020 \AA ; 2.7 \AA ; 0.02-0.03 \AA \mathrm{pix}^{-1}$ & $900 \mathrm{~s}$ \\
\hline $03 /[24,27,29] / 01 ; 02 /[13,15,16,21] / 03$ & CTIO 1.5m (RC Spectrograph) & 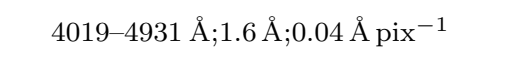 & $300-400$ s \\
\hline $03 / 13 / 03 ; 06 / 11 / 06$ & CTIO 1.5m (RC Spectrograph) & $4019-4931 \AA$; $1.6 \AA ; 0.04 \AA \mathrm{pix}^{-1}$ & $300-400 \mathrm{~s}$ \\
\hline $03 /[24,27,29] / 01 ; 02 /[13,15,16,21] / 03$ & CTIO 1.5m (RC Spectrograph) & $6012-7332 \AA ; 3.1 \AA ; 0.04 \AA \mathrm{pix}^{-1}$ & $60 \mathrm{~s}$ \\
\hline $03 / 13 / 03 ; 06 / 11 / 06$ & CTIO 1.5m (RC Spectrograph) & $6012-7332 \AA ; 3.1 \AA ; 0.04 \AA \mathrm{pix}^{-1}$ & $60 \mathrm{~s}$ \\
\hline $01 / 21 / 07$ & CTIO 1.5m (RC Spectrograph) & $3400-6850 \AA ; 11.6 \AA ; 0.12 \AA \mathrm{pix}^{-1}$ & $300 \mathrm{~s}$ \\
\hline
\end{tabular}

${ }^{a}$ RMS was not recorded 
Table 4. Stellar parameters and abundances from spectral synthesis

\begin{tabular}{lll}
\hline \hline \multicolumn{1}{c}{ Quantity } & Primary Star & Secondary Star \\
\hline$T_{\text {eff }}(\mathrm{K})$ & $9250 \pm 250$ & $9150 \pm 250$ \\
$\log g$ & $4.0 \pm 0.3$ & $3.5 \pm 0.3$ \\
{$[\mathrm{Fe} / \mathrm{H}]$} & $+0.01 \pm 0.14$ & $0.00 \pm 0.14$ \\
{$[\mathrm{Ti} / \mathrm{H}]$} & $+0.59 \pm 0.15$ & $+0.44 \pm 0.16$ \\
{$[\mathrm{Mg} / \mathrm{H}]$} & $+0.34 \pm 0.20$ & $+0.41 \pm 0.20$ \\
$v \sin i\left(\mathrm{~km} \mathrm{~s}^{-1}\right)$ & $17 \pm 3$ & $<5$ \\
$\xi_{\mathrm{t}}\left(\mathrm{km} \mathrm{s}{ }^{-1}\right)$ & $3.2 \pm 0.2$ & $2.6 \pm 0.2$ \\
Luminosity fraction & $0.50 \pm 0.02$ & $0.50 \pm 0.02$ \\
\hline
\end{tabular}


Table 5. Radial velocities $\left(\mathrm{km} \mathrm{s}^{-1}\right)$

\begin{tabular}{|c|c|c|c|c|c|c|c|}
\hline $\begin{array}{l}\text { HJD } \\
(24+)\end{array}$ & \multicolumn{7}{|c|}{$(24+)$} \\
\hline \multicolumn{4}{|c|}{ AAT echelle UCLES } & 52005.18208 & $102.9 \pm 2.7$ & $-113.7 \pm 2.9$ & .226 \\
\hline 51686.00921 & $100.66 \pm 0.12$ & $-115.32 \pm 0.21$ & .219 & 52005.20053 & $103.5 \pm 2.9$ & $-110.4 \pm 3.0$ & .230 \\
\hline 51686.07438 & $101.32 \pm 0.14$ & $-116.75 \pm 0.22$ & .232 & 52005.21536 & $103.9 \pm 2.9$ & $-111.1 \pm 3.1$ & .233 \\
\hline 51687.02352 & $37.24 \pm 0.14$ & $-52.69 \pm 0.24$ & .426 & 52005.23149 & $103.2 \pm 2.7$ & $-112.3 \pm 2.8$ & .236 \\
\hline 51687.21123 & $15.96 \pm 0.26$ & $-28.56 \pm 0.35$ & .464 & 52005.24632 & $104.6 \pm 2.8$ & $-112.3 \pm 3.0$ & .239 \\
\hline 51687.84689 & $-69.21 \pm 0.25$ & $51.33 \pm 0.43$ & .593 & 52005.26550 & $107.2 \pm 2.8$ & $-111.6 \pm 2.9$ & .243 \\
\hline 51687.91204 & $-77.22 \pm 0.16$ & $60.24 \pm 0.25$ & .607 & 52005.28034 & $106.6 \pm 2.8$ & $-112.9 \pm 2.9$ & .246 \\
\hline 51688.17285 & $-100.57 \pm 0.24$ & $84.98 \pm 0.38$ & .660 & 52005.29655 & $103.4 \pm 2.8$ & $-114.5 \pm 2.9$ & .249 \\
\hline \multicolumn{4}{|c|}{ AAT mid-resolution RGO } & 52005.31138 & $103.1 \pm 2.8$ & $-112.9 \pm 3.0$ & .252 \\
\hline 52003.09624 & $-104.9 \pm 5.8$ & & .801 & \multicolumn{4}{|c|}{ NTT low resolution EMMI } \\
\hline 52003.16759 & $-104.9 \pm 3.0$ & $96.3 \pm 5.0$ & .816 & 49827.817370 & $-159.7 \pm 4.9$ & $58.6 \pm 5.3$ & .757 \\
\hline 52003.18666 & & $83.1 \pm 8.0$ & .819 & 49827.865438 & $-143.5 \pm 6.1$ & $70.3 \pm 6.9$ & .767 \\
\hline 52003.19897 & $-107.6 \pm 4.6$ & $92.8 \pm 4.6$ & .822 & 49827.916331 & $-139.4 \pm 6.8$ & $66.1 \pm 7.6$ & .777 \\
\hline 52003.22556 & $-103.2 \pm 4.4$ & $91.5 \pm 4.4$ & .827 & \multicolumn{4}{|c|}{ SAAO mid-resolution RPCS } \\
\hline 52003.24120 & $-100.1 \pm 7.6$ & $82.8 \pm 8.1$ & .831 & 49861.40278 & $-90.4 \pm 8.9$ & $30.0 \pm 11.1$ & .598 \\
\hline 52003.25740 & $-101.4 \pm 2.2$ & $86.6 \pm 4.2$ & .834 & 49861.41874 & $-85.4 \pm 12.0$ & $34.5 \pm 10.9$ & .601 \\
\hline 52003.27586 & $-104.7 \pm 5.0$ & $87.4 \pm 4.1$ & .838 & 49861.43428 & $-97.0 \pm 10.2$ & $32.7 \pm 11.6$ & .604 \\
\hline 52004.92234 & $92.7 \pm 2.9$ & $-99.7 \pm 3.0$ & .173 & 49861.44994 & $-93.2 \pm 5.8$ & $43.6 \pm 9.0$ & .607 \\
\hline 52004.93856 & $94.0 \pm 2.9$ & $-98.6 \pm 3.0$ & .176 & 49861.56414 & $-105.7 \pm 7.8$ & $66.0 \pm 1.9$ & .630 \\
\hline 52004.95863 & $96.0 \pm 2.8$ & $-102.9 \pm 2.9$ & .180 & 49861.57973 & $-79.3 \pm 15.7$ & $77.1 \pm 12.4$ & .634 \\
\hline 52004.97347 & $95.1 \pm 3.0$ & $-99.9 \pm 3.2$ & .183 & 49861.59529 & $-92.3 \pm 11.3$ & $71.4 \pm 11.2$ & .637 \\
\hline 52004.99521 & $100.5 \pm 2.7$ & $-99.2 \pm 2.8$ & .188 & 49861.61082 & $-92.4 \pm 13.5$ & & .640 \\
\hline 52005.01723 & $99.8 \pm 2.6$ & $-103.3 \pm 2.8$ & .192 & 49862.21732 & $-124.2 \pm 9.1$ & $95.6 \pm 5.2$ & .764 \\
\hline 52005.16725 & $104.3 \pm 2.7$ & $-111.6 \pm 2.8$ & .223 & 49862.23307 & $-122.8 \pm 9.42$ & $82.5 \pm 8.2$ & .767 \\
\hline
\end{tabular}


Table 5. Radial velocities $\left(\mathrm{km} \mathrm{s}^{-1}\right)$; con't

\begin{tabular}{|c|c|c|c|c|c|c|c|}
\hline $\begin{array}{l}\text { HJD } \\
(24+)\end{array}$ & Secondary & Primary & Phase & $\begin{array}{l}\text { HJD } \\
(24+)\end{array}$ & Secondary & Primary & Phase \\
\hline \multicolumn{8}{|c|}{ SAAO mid-resolution RPCS } \\
\hline 49862.24861 & $-131.7 \pm 8.87$ & $87.7 \pm 8.1$ & .770 & 49867.22227 & $-128.6 \pm 23.2$ & $79.0 \pm 13.5$ & .783 \\
\hline 49862.26419 & $-144.2 \pm 9.5$ & $92.7 \pm 3.8$ & .773 & 49867.23781 & & $79.4 \pm 10.0$ & .786 \\
\hline 49862.38420 & $-135.2 \pm 18.7$ & $88.6 \pm 12.5$ & .797 & 49867.28447 & $-127.3 \pm 13.0$ & & .796 \\
\hline 49862.39977 & $-120.5 \pm 11.3$ & $92.1 \pm 10.0$ & .801 & 49867.30002 & $-123.4 \pm 9.2$ & & .799 \\
\hline 49862.41619 & $-117.4 \pm 12.5$ & $82.8 \pm 8.6$ & .804 & 49867.31561 & $-147.5 \pm 14.0$ & & .802 \\
\hline 49862.43180 & $-118.1 \pm 7.7$ & $88.7 \pm 9.5$ & .807 & 49867.42737 & $-126.9 \pm 11.9$ & $65.0 \pm 13.6$ & .825 \\
\hline 49862.54706 & $-126.7 \pm 10.5$ & $85.0 \pm 10.5$ & .831 & 49867.44294 & $-135.1 \pm 15.3$ & $64.5 \pm 12.3$ & .828 \\
\hline 49862.56327 & $-105.2 \pm 15.5$ & $89.0 \pm 23.2$ & .834 & 49867.45848 & $-122.9 \pm 14.2$ & $74.2 \pm 9.2$ & .831 \\
\hline 49862.57945 & $-111.5 \pm 7.6$ & $80.3 \pm 13.8$ & .837 & 49867.47402 & $-126.4 \pm 12.2$ & $79.1 \pm 19.7$ & .834 \\
\hline 49866.25795 & $-102.8 \pm 7.9$ & $34.1 \pm 11.6$ & .586 & 49864.33839 & $79.5 \pm 8.3$ & $-127.4 \pm 8.9$ & .196 \\
\hline 49866.27357 & $-73.0 \pm 20.9$ & $19.7 \pm 15.3$ & .590 & 49864.37070 & $65.1 \pm 7.8$ & $-135.6 \pm 12.9$ & .202 \\
\hline 49866.28914 & $-79.4 \pm 13.0$ & $27.8 \pm 17.3$ & .593 & 49864.38793 & $55.2 \pm 12.3$ & $-122.8 \pm 29.1$ & .206 \\
\hline 49866.30470 & $-100.8 \pm 16.7$ & $29.1 \pm 10.7$ & .596 & 49864.40409 & $64.1 \pm 11.9$ & $-132.6 \pm 10.9$ & .209 \\
\hline 49866.38020 & $-103.0 \pm 16.8$ & & .611 & 49864.41963 & $78.9 \pm 8.7$ & $-142.5 \pm 15.0$ & .212 \\
\hline 49866.44475 & $-111.6 \pm 11.1$ & $68.0 \pm 17.2$ & .625 & 49865.40475 & $51.9 \pm 5.5$ & $-87.2 \pm 10.0$ & .413 \\
\hline 49866.46029 & $-104.5 \pm 6.4$ & & .628 & 49865.42029 & $42.9 \pm 11.1$ & $-79.9 \pm 19.5$ & .416 \\
\hline 49866.47583 & $-103.9 \pm 12.9$ & & .631 & 49865.45146 & & $-74.0 \pm 17.5$ & .422 \\
\hline
\end{tabular}


Table 6. Orbital parameters of NSV 19992 from AAT echelle data only

\begin{tabular}{lc}
\hline \hline \multicolumn{1}{c}{ Parameter } & Value \\
\hline & \\
$q \equiv M_{2} / M_{1}{ }^{\mathrm{a}}$ & $0.9921 \pm 0.0097$ \\
$a \sin i\left[\mathrm{R}_{\odot}\right]$ & $21.096 \pm 0.147$ \\
$e$ & $0.992 \pm 0.004$ \\
$M_{\mathrm{tot}} \sin ^{3} i\left[\mathrm{M}_{\odot}\right]$ & $5.232 \pm 0.070$ \\
$K_{1}$ & $109.37 \pm 0.50 \mathrm{~km} \mathrm{~s}^{-1}$ \\
$K_{2}$ & $110.23 \pm 0.50 \mathrm{~km} \mathrm{~s}^{-1}$ \\
$M_{1} \sin ^{3} i$ & $2.616 \pm 0.037 \mathrm{M}_{\odot}$ \\
$M_{2} \sin ^{3} i$ & $2.596 \pm 0.037 \mathrm{M}_{\odot}$ \\
$\mathrm{P}$ & $4.9098505 \pm 0.0000020 \mathrm{~d}$ \\
$\mathrm{~T}_{0}$ & $2450668.5915 \pm 0.0010 \mathrm{~d}$ \\
\hline
\end{tabular}

${ }^{\mathrm{a}} 1=$ primary; $2=$ secondary

Table 7. Systemic Velocity of NSV 19992 from each Epoch of Radial-Velocity Data

\begin{tabular}{lrc}
\hline \hline Observing Run & Mean Epoch & \multicolumn{1}{c}{$v_{\gamma}\left[\mathrm{km} \mathrm{s}^{-1}\right]$} \\
\hline NTT low res. & 1995.30 & $-43.08 \pm 2.72$ \\
SAAO mid res. & 1995.40 & $-21.36 \pm 1.13$ \\
AAT echelle & 2000.39 & $-7.82 \pm 0.41$ \\
AAT mid res. & 2001.25 & $-4.08 \pm 0.36$ \\
\hline
\end{tabular}


Table 8. Orbital parameters of NSV 19992 from fit to all radial velocity data

\begin{tabular}{|c|c|}
\hline Parameter & Value \\
\hline$q \equiv M_{2} / M_{1}$ & $0.992 \pm 0.004$ \\
\hline$a \sin i\left[\mathrm{R}_{\odot}\right]$ & $21.066 \pm 0.050$ \\
\hline$e$ & $0.023 \pm 0.011^{\mathrm{a}}$ \\
\hline$M_{\text {tot }} \sin ^{3} i\left[\mathrm{M}_{\odot}\right]$ & $5.210 \pm 0.037$ \\
\hline
\end{tabular}

${ }^{a}$ Eccentricity fixed at zero for calculations-see $\S 6.2$ 
Table 9. Physical parameters of the components of NSV 19992

\begin{tabular}{lc}
\hline \hline \multicolumn{1}{c}{ Parameter } & Value \\
\hline$i\left[^{\circ}\right]$ & $81.5 \pm 0.1$ \\
$M_{1}\left[M_{\odot}\right]$ & $2.704 \pm 0.038$ \\
$M_{2}\left[M_{\odot}\right]$ & $2.683 \pm 0.038$ \\
$T_{1} / T_{2}$ & $1.0016 \pm 0.0018$ \\
$R_{1}+R_{2}\left[R_{\odot}\right]$ & $8.195 \pm 0.046$ \\
$R_{1} / R_{2}{ }^{\mathrm{a}}$ & $0.996 \pm 0.029$ \\
\hline
\end{tabular}

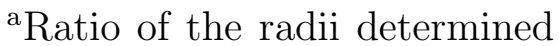
from the temperature and the spectroscopically determined luminosity ratio $L_{1} / L_{2}=1.000 \pm$ 0.057 